Leadership individuel et leadership collectif : une identification des leaders de marché et de leurs performances économiques grâce à l'analyse des réseaux sociaux

Thomas Dallery, Fabien Éloire et Jordan Melmiès

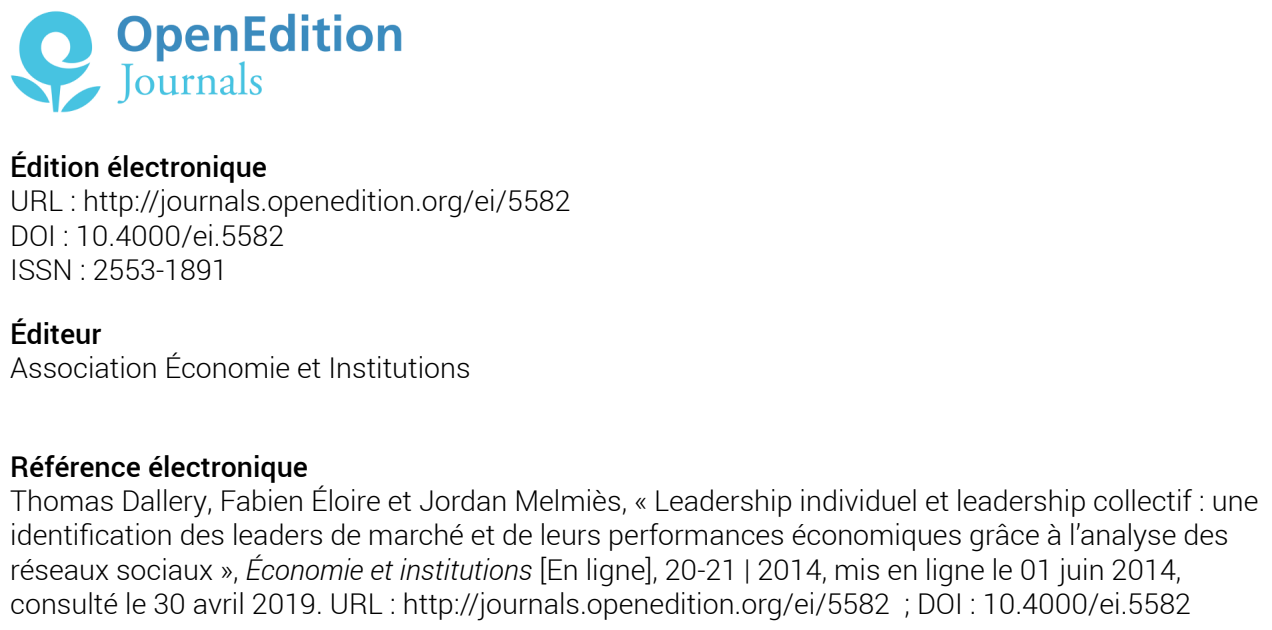

Ce document a été généré automatiquement le 30 avril 2019.

Revue Économie et institutions 


\title{
Leadership individuel et leadership collectif : une identification des leaders de marché et de leurs performances économiques grâce à l'analyse des réseaux sociaux
}

\author{
Thomas Dallery, Fabien Éloire et Jordan Melmiès
}

\section{Introduction}

Fruit de la rencontre entre la sociologie économique structurale et la théorie postkeynésienne, cet article traite de la question de la formation des prix sur un marché. Partant de notre camp de base post-keynésien, nous enrichissons l'analyse postkeynésienne des marchés, d'une part en contribuant à revisiter l'une des théories postkeynésiennes du prix grâce à l'analyse des réseaux sociaux, et d'autre part en soulignant l'importance des structures sociales à la base des phénomènes de concurrence sur un marché. Nous proposons de tester l'une des théories post-keynésiennes de la formation des prix : le price leadership. Selon cette théorie, et conformément aux théories plus larges dites du cost-plus (Lavoie, 2004), le prix est obtenu en ajoutant une marge unitaire de profit à une mesure des coûts unitaires. Là où la théorie du price leadership trouve son originalité, c'est dans la manière dont émergent ces deux éléments de coûts et de marge. Selon cette théorie, un marché serait divisé entre des entreprises leaders et des entreprises suiveuses, dominées. Les leaders ont une technologie supposée efficace en termes de coûts, et ils fixent ensuite une marge satisfaisante permettant de répondre à différents objectifs (autofinancement de l'investissement notamment). Le prix est donc déterminé par les leaders. Les entreprises dominées doivent alors suivre ce prix (concurrence par les prix), et compte tenu de leur capacité plus ou moins grande à contrôler leurs coûts, elles devront se satisfaire d'une marge de profit plus ou moins 
importante. Si les coûts ne sont pas suffisamment bien contrôlés, la théorie du price leadership prédit que les entreprises dominées réaliseront une marge trop faible pour survivre (notamment $\mathrm{du}$ fait de l'incapacité à assurer l'autofinancement de l'investissement). ${ }^{1}$

2 En plus de la question de la formation des prix, l'article aborde donc la délicate question du leadership sur un marché. Pour confronter la théorie du price leadership à nos données², nous avons été confrontés à une question de définition: qu'est-ce qu'un leader? La théorie post-keynésienne est floue sur le sujet. La définition renvoie tantôt à une grande part de marché, tantôt à une efficacité technologique, tantôt à un effet d'impulsion (est leader celui qui joue en premier...). Devant cette imprécision, nous avons cherché une définition plus claire, mais restant cohérente avec le cadre théorique du price leadership : est leader celui qui est regardé par les autres. Les outils de la sociologie des réseaux permettent alors d'identifier les leaders.

Parés de cette définition relationnelle du leadership, nous procédons ensuite à un test visant à valider ou invalider la théorie du price leadership : les leaders font-ils des marges de profit supérieures aux autres? Les résultats présentés ici suggèrent que les prédictions de la théorie sont confirmées, et ce, au niveau individuel et collectif, dans une majorité de cas. Quand les leaders ne réalisent pas les meilleures marges de profit, nous montrons par ailleurs, que cela s'explique, d'une part parce que le leadership relationnel n'est pas unidimensionnel (la centralité relationnelle ne suffit pas pour comprendre la performance économique lorsque la concurrence ne s'exerce plus sur le terrain économique mais sur le terrain symbolique), et d'autre part parce que les entreprises dominées poursuivent des stratégies de report de contrainte, notamment sur la qualité.

L'article se décompose comme suit. La première partie montre comment, en mêlant l'économie post-keynésienne (Lavoie, 2004) et la sociologie économique structurale (White, 1981), il est possible d'enrichir la compréhension du prix sur un marché, et comment en particulier la théorie du price leadership peut trouver à être élargie. La deuxième partie développe les tests empiriques qui ont été menés pour valider nos apports théoriques. Après avoir présenté les données d'enquête, elle montre l'apport de l'analyse de réseaux sociaux pour identifier les leaders, en mobilisant d'abord les scores de centralité, puis la technique du blockmodelling.

\section{Une approche hétérodoxe du prix}

Dans cette première partie de l'article, nous nous situons dans le moment théorique où nous montrons les gains liés au dialogue entre théorie post-keynésienne et sociologie économique structurale. Dans un premier paragraphe, nous allons d'abord présenter l'approche générale du prix chez les Post-Keynésiens. Nous verrons également en quoi notre théorie des reports de contrainte entre parties prenantes converge avec la sociologie économique structurale de White. Dans un second paragraphe, nous traiterons plus spécifiquement d'une théorie particulière au sein des approches post-keynésiennes du prix en présentant la théorie du price leadership, puis nous montrerons comment il est possible d'aboutir à une théorie davantage élaborée grâce aux outils de l'analyse des réseaux sociaux. C'est seulement dans la seconde partie que nous présenterons les résultats de l'enquête de terrain. 


\subsection{Le prix chez les Post-Keynésiens}

6 Pour schématiser sans doute avec excès, la firme n'a à prendre "que " ces quelques décisions : i) elle doit décider de la qualité du produit qu'elle va offrir, ii) de la manière de produire ce bien, iii) de la quantité qu'elle va en offrir, et finalement iv) du prix auquel elle désire vendre sa production (tout cela étant lié à des décisions d'investir par des relations de codétermination). Les réponses à l'ensemble de ces questions sont largement dépendantes du chemin parcouru par la firme. ${ }^{3}$

7 La décision en matière de fixation de prix est inséparablement liée au " projet initial de l'entreprise » (i.e., un ensemble de critères: produit, qualité, capacité de production, localisation géographique,...) et à la stratégie mise en œuvre pour le réaliser. Dans cette perspective, le prix reflète moins la rareté, la singularité de la production, que les coûts de réalisation qu'elle engage. Autrement dit, les prix sont fixés avant toute transaction, ils n'ont pas vocation à équilibrer l'offre et la demande de marché ni à maximiser les profits, mais à fournir les fonds nécessaires à la reproduction dans le temps de la firme et de ses objectifs. Les Post-Keynésiens se basent une théorie de la fixation des prix adaptée à cette situation qui repose sur « le principe du calcul de coût ou cost-plus » (Lavoie, 2004, p. 44) : le prix résulte de l'ajout d'une marge de profit à une mesure du coût unitaire, par exemple le coût unitaire direct ${ }^{4}$ :

$$
\mathrm{p}=(1+\theta) \text { CUD (1) }
$$

8

Le fait de poser que le prix se compose des deux éléments de coût et de marge revient à considérer que sa fixation est conditionnée à trois types de rapports interdépendants avec, premièrement les fournisseurs, deuxièmement les acheteurs, troisièmement les pairs/concurrents, qui renvoient aux trois dimensions du marché tripartite de White (1981) : 
Figure 1. Le marché tripartite dans une économie de production selon White

\section{Fournisseurs}

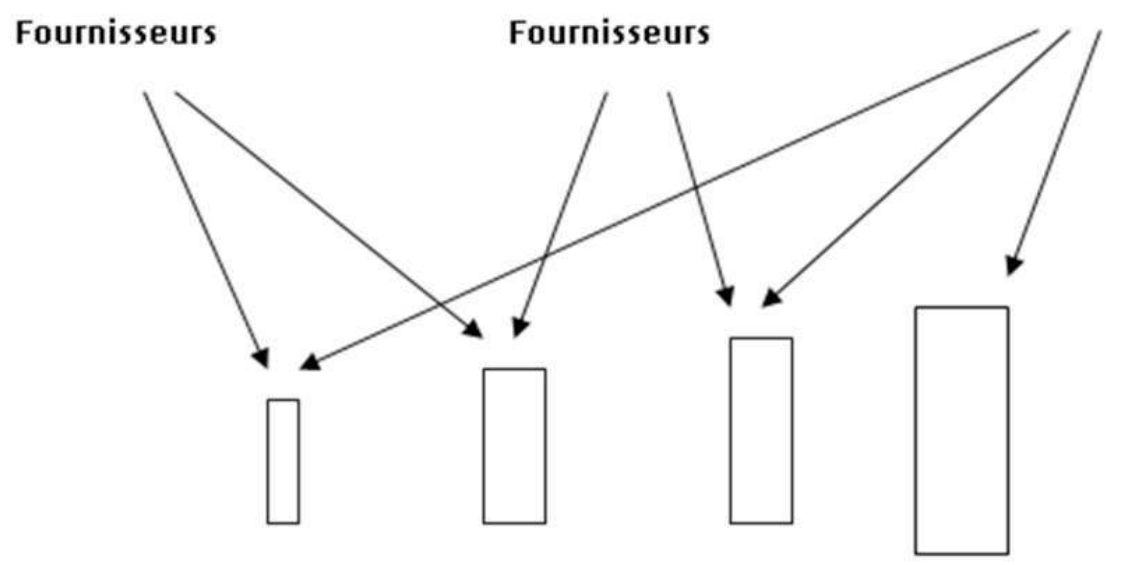

(entreprises classées par ordre de qualité)

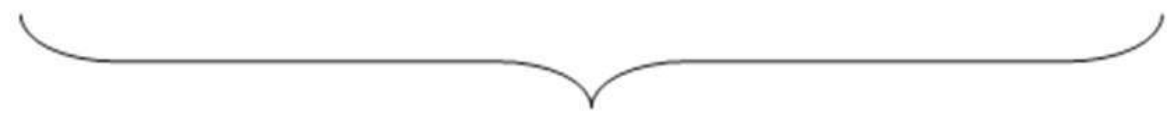

\section{Clientagrégé}

Source : White, 2002, p. 6

Les trois types de rapports interdépendants sont :

- le rapport avec les fournisseurs/salariés, dont dépend le volume des coûts (matières premières et masse salariale principalement). Plus le pouvoir économique ou symbolique de négociation est important plus les coûts pourront être réduits (par rapport aux concurrents) et plus les prix pourront être fixés bas ou plus les marges pourront être élevées. Tournés vers le volume, les entrepreneurs auront des relations privilégiées avec leurs fournisseurs et pourront escompter des remises du fait des quantités commandées. De même, les relations de l'entrepreneur avec ses salariés seraient marquées par une grande volonté de maîtrise des coûts salariaux : les salariés de ces firmes ne se verraient pas offrir des salaires très intéressants et ils se verraient souvent usés par le métier et découragés par la rémunération, de sorte qu'on constaterait un fort taux de turnover dans la main d'œuvre ${ }^{5}$. Le rapport avec les fournisseurs/salariés permet surtout de prendre en compte l'aspect essentiel de la qualité : plus une production est de bonne qualité, plus cela suppose des coûts élevés (main d'œuvre qualifiée, produits intermédiaires singuliers,...).

- le rapport avec les acheteurs, dont dépend la sanction positive ou négative de la demande agrégée ; ainsi, le taux d'utilisation de ses capacités de production indique à l'entrepreneur si la niche de qualité qu'il occupe et la marge de profit qu'il a déterminée en accord avec son projet d'entreprise sont viables ou non ; et, à court terme, la demande ne détermine pas tant le prix (la marge) que le taux d'utilisation des capacités. Aussi, si la sanction d'une sousutilisation des capacités peut inciter l'entrepreneur à réviser sa politique de marge et de prix à moyen terme, il n'en reste pas moins que ses marges de manœuvre sont restreintes et que la sanction de la demande porte plus sur le projet d'entreprise dans son ensemble que sur ses prix. Pour le dire autrement, la demande ne détermine pas la marge de profit de l'entreprise, mais le montant de ses profits. 
- le rapport avec les pairs/concurrents, dont dépend l'espace des possibles, c'est-à-dire la fourchette de prix au sein de laquelle il est raisonnable de se situer eu égard à la production proposée. White $(1981,2002)$ insiste sur le fait que les producteurs construisent collectivement l'échelle de qualité au sein de laquelle ils se distribuent ensuite. La construction collective de la qualité est liée, chez White, au fait que les producteurs s'observent entre eux. Ils observent la quantité de produits vendus et la recette réalisée pour cette quantité. Le verbe « observer " prend ici un sens large : on s'observe certes pour mieux s'imiter, se copier ; mais on le fait aussi pour mieux se démarquer, se différencier des autres, cultiver son originalité et se positionner dans l'interface. L'observation crée donc le conformisme, l'inertie, la reproduction; mais est tout autant porteuse d'innovation, de nouveauté, de changement, de singularité (Karpik, 2007).

Dès lors, il apparaît clairement que les rapports qu'entretient la firme avec les trois différentes parties (acheteurs, fournisseurs, pairs/concurrents) sont mutuellement conflictuels. Il faut en quelque sorte choisir lequel de ces rapports/objectifs doit primer sur les deux autres (Dallery et van Treeck, 2010). Si la satisfaction des acheteurs est le premier objectif de l'entrepreneur, il devra s'efforcer d'offrir la meilleure qualité au meilleur prix. Cela supposera d'obtenir une qualité en accord avec son prix, et d'augmenter la qualité pour un prix donné (augmentation des coûts et réduction de la marge). Si l'objectif premier de l'entrepreneur est la réalisation de la qualité, et la reconnaissance de ses pairs, il favorisera le rapport avec ses fournisseurs (de matières premières et de travail), quitte à ce que cela se fasse au détriment de l'acheteur (prix élevé eu égard à la qualité jugée par le client agrégé), ou de lui-même (marge faible pour coller au jugement de l'acheteur agrégé). Si, enfin, l'entrepreneur entre dans une logique de compétition dans son rapport avec ses pairs/concurrents, cela pourra se faire au détriment des fournisseurs (pression sur les prix transférée sur les coûts ; pression sur les salaires et les conditions de travail) et, à l'avantage de l'acheteur éventuellement (logique contradictoire entre baisse du prix et baisse de la qualité).

La résolution concrète de ces conflits supposera d'atteindre un compromis entre les différentes parties prenantes et les tensions qu'elles génèrent sur les différentes composantes de prix, de marge et de qualité. Le prix, comme rapport social, traduit plus un équilibre des rapports de force (combinant le produit, la qualité, les capacités de production, la localisation géographique,...) qu'un équilibre des plans (offre et demande).

\subsection{La théorie du price leadership}

Dans la section précédente, nous avons présenté l'approche post-keynésienne du prix et de sa décomposition entre éléments de marge et de coûts (la théorie dite du cost-plus), et nous avons montré comment cette théorie du prix collait avec la représentation du marché tripartite (White, 1981) et avec la théorie des reports de contrainte entre parties prenantes (Dallery et van Treeck, 2010). Cette approche post-keynésienne du prix a cependant laissé en suspens au moins une question essentielle : à quel niveau exactement se fixent prix et marges?

L'une des réponses souvent avancées par les Post-Keynésiens est que la marge se fixe au niveau où elle permet la reproduction dans le temps de l'entreprise, grâce au financement de l'investissement ${ }^{6}$. Cette réponse semble parfaitement valable pour une firme prise isolément. Mais, qu'en est-il lorsque l'on s'intéresse à un ensemble de firmes sur un 
marché? Indéniablement, sur un marché donné, il règne une forme d'harmonie ou d'homogénéité des prix, la concurrence poussant plutôt à une uniformisation des prix.

Mais est-ce alors à dire que toutes les firmes ont les mêmes prix parce qu'elles ont les mêmes coûts et les mêmes marges ? Bien évidemment, une uniformité des prix ne préjuge pas d'une uniformité des coûts et des marges, à moins d'y voir l'incarnation dans le réel de la théorie de l'agent représentatif. Des firmes différentes ont des projets d'entreprise, donc des coûts, différents, et par conséquent, elles n'auront pas les mêmes capacités à dégager des marges de profit sensiblement identiques pour un prix donné. Ici, nous allons présenter une théorie particulière de la formation des prix chez les Post-Keynésiens : la théorie du price leadership. Selon cette théorie, la marge sert toujours à la reproduction dans le temps de la firme. Mais dans une situation où plusieurs firmes se font concurrence, un prix peut s'imposer et contraindre certaines firmes à comprimer leurs marges, à défaut de pouvoir comprimer leurs coûts (par exemple en réduisant la qualité), pour s'aligner sur ce prix « directeur ».

Où se fixe alors ce prix de leadership, ce prix « directeur "? La théorie du price leadership aboutit à la conclusion qu'une firme particulière (ou un ensemble restreint de firmes) peut être en mesure d'imposer "son" prix aux autres firmes. Cette firme leader a la capacité d'imposer son prix comme une référence pour les autres participants au marché. Elle a un projet d'entreprise qui détermine ses coûts; et une stratégie de reproduction dans le temps qui détermine ses projets d'investissement et la marge de profit nécessaire. De ces coûts et de ces marges de profit découle enfin un prix pour l'entreprise leader.

Les leaders établissent «librement » leur prix, et la marge qui en découle les satisfait pleinement compte-tenu de leurs autres contraintes. Dans cette théorie, les entreprises s'observent et se suivent (Lavoie, 2004, p. 50). Une hiérarchie est établie entre ces entreprises leaders et des entreprises dominées ou suiveuses. Les entreprises dominées sont par contre forcées de suivre le mouvement initié par les leaders et de s'aligner sur leur prix, ce qui peut conduire à rogner sur leurs marges pour suivre le prix des leaders. ${ }^{7}$ La survie à long terme des entreprises dominées dépendra alors de leur capacité à améliorer la maîtrise de leurs coûts (par exemple grâce à une baisse de la qualité) afin de mieux coller à la marge des entreprises leaders. ${ }^{8}$

17 Mais survient alors la question : pourquoi une firme est-elle leader? La réponse à cette question n'est pas univoque parmi les Post-Keynésiens. Rappelons qu'initialement, les Post-Keynésiens ont été reconnus comme les théoriciens de la concurrence imparfaite, que ce soit par les premiers travaux de Joan Robinson (Robinson, 1933) ou par les travaux de Michal Kalecki sur le degré de monopole (Kalecki, 1954). Cette théorie de la concurrence imparfaite fournit à elle seule une explication de la marge de profit des entreprises : l'ampleur de la marge de profit s'explique par le degré d'imperfection de la concurrence, ou encore le degré de monopole.

La théorie du price leadership se comprend aussi dans ce cadre originel de la concurrence imparfaite pour les Post-Keynésiens : la distinction entre firme leader et firmes suiveuses est initialement à replacer dans un environnement de marchés où seuls quelques offreurs s'affrontent. La firme leader est alors celle qui a la plus grosse part de marché (Eichner, 1976) ${ }^{9}$. Cette définition du leadership ne nous semble cependant pas convaincante : d'une part, elle semble peu pertinente eu égard à la réalité observée des oligopoles (les plus grandes entreprises ne sont pas forcément celles qui font le plus de marge); d'autre part, elle n'est qu'une théorie statique qui ne questionne que peu l'origine du leadership. 
19 Par ailleurs, il semble bien que le cadre de référence de la concurrence imparfaite soit incontournable pour raisonner en termes de parts de marché, de sorte que la théorie du price leadership ne s'appliquerait que dans le cadre restreint de la concurrence imparfaite. Mais les auteurs mêmes qui étaient à l'origine de cette théorie de la concurrence imparfaite ont fini par douter de sa pertinence et à la remettre en cause (Melmiès, 2009). Si la concurrence imparfaite est bien le berceau des idées post-keynésiennes en matière de prix, elle ne constitue qu'un point de départ à l'analyse post-keynésienne des prix et de la concurrence, mais pas un point d'arrivée (Melmiès, 2011). Aussi, nous proposons ici de dépasser le caractère imparfait de la concurrence qui semble indissociable de la théorie du price leadership, afin de montrer que les phénomènes de leadership dans la détermination des prix existent et ce, même dans un cadre se rapprochant de la concurrence pure et parfaite avec un nombre élevé de producteurs. C'est donc là le premier apport de notre travail que de vouloir défendre l'idée selon laquelle les PostKeynésiens ne doivent pas se limiter à des analyses dans un cadre de concurrence imparfaite, mais doivent au contraire investir le champ de la concurrence parfaite. Dans la seconde partie de l'article, nous proposerons donc d'appliquer ce cadre théorique du price leadership à un environnement de marché se rapprochant de la concurrence pure et parfaite avec un grand nombre de firmes, en réalisant des tests empiriques sur le marché de la restauration au sein de la métropole lilloise.

De plus, compte-tenu de notre insatisfaction liée aux facteurs déterminant traditionnellement le leadership dans l'analyse post-keynésienne, nous proposons des fondements que nous pensons plus solides au leadership sur un marché : une firme sera leader grâce à la possession d'un capital symbolique, culturel ou économique spécifique (Bourdieu, 2000). C'est bien la reconnaissance par les autres de ce statut de leader, sur des bases objectives et/ou subjectives, qui permet à la firme leader de se comporter comme tel et, en particulier, d'imposer son prix comme référence aux autres. Cette reconnaissance du leadership suppose alors qu'est leader celui qui est regardé.

21 L'analyse des réseaux sociaux fournit les outils pour identifier ces leaders de marché en isolant les firmes qui sont regardées par les autres firmes du marché. La dichotomie leader / suiveur sera donc arbitrée par la dichotomie observé / observateur. Selon la théorie du price leadership, les leaders, ainsi identifiés par leur centralité relationnelle, devraient exhiber une meilleure marge de profit. C'est bien à ce test que nous allons procéder dans la suite de cet article. La théorie du price leadership prédit que les leaders ont une marge de profit supérieure. Nous supposons, pour notre part, que les leaders seront identifiés par le côté relationnel (être cité par les autres fait de vous un leader), et c'est seulement dans un second temps, avec cette conception du leadership, que nous testerons la théorie du price leadership, à savoir : les leaders relationnels ont-ils des marges de profit supérieures aux autres?

\section{Le marché des restaurateurs lillois comme lieu d'expérimentation de la théorie du price leadership}

Dans cette seconde partie, nous passons du moment théorique à l'application empirique. Dans un premier temps, nous présentons l'enquête de terrain qui nous a permis d'obtenir les données grâce auxquelles nous réaliserons nos tests. Dans un deuxième temps, nous introduisons une première mesure du leadership: la centralité relationnelle "brute" (le 
fait d'être cité par les autres). Dans un troisième temps, avec la technique du blockmodelling, nous montrons que la structuration du marché en champ doit être intégrée à l'analyse pour réellement identifier les leaders au sein d'un marché, désormais divisé en blocks homogènes sur le plan relationnel.

\subsection{Le marché des restaurateurs lillois comme réseau social}

Afin de donner une consistance empirique à notre réflexion sur la théorie du price leadership et sur la notion de leadership économique, nous avons recours aux données d'une enquête de terrain réalisée auprès d'une population de restaurateurs indépendants. Ces données ont été construites dans le cadre d'une étude de sociologie économique structurale qui a mobilisé l'analyse des réseaux sociaux. Ayant décrit et analysé ailleurs les dimensions économique (Dallery, Eloire, Melmiès, 2009) et sociologique (Dallery, Eloire, Melmiès, 2010) de ce marché, nous nous intéressons ici à sa dimension relationnelle. Nous examinons, plus particulièrement, le lien entre le leadership économique, tel que nous venons de le définir, c'est-à-dire d'un point de vue relationnel, et la performance économique, que nous mesurons par le taux de marge nette des entreprises enquêtées ${ }^{10}$.

L'utilisation des outils d'analyse de réseaux (Wasserman, Faust, 1994 ; Lazega, 1998) permet à la sociologie économique structurale de proposer une vision relativement contre-intuitive du marché et de la concurrence (Eloire, 2010). En insistant sur les interdépendances de ressources sociales, c'est-à-dire sur les transferts et échanges de type non-économique entre les entrepreneurs, elle met l'accent sur l'existence de formes de coopération (toujours plus ou moins temporaires et limitées) qui sont susceptibles de se développer dans les milieux les plus concurrentiels ${ }^{11}$. Le milieu des restaurateurs est de ceux-là : le choix d'une telle population comme objet pour une étude de réseaux ne va, au départ, pas de soi, puisqu'il s'agit de chefs d'entreprise juridiquement indépendants, et économiquement concurrents au sein d'un même marché. Pourtant, ces restaurateurs n'en constituent pas moins un milieu professionnel à la fois ouvert, car il n'est pas nécessaire de posséder un diplôme en hôtellerie-restauration pour s'installer comme restaurateur, et organisé, car possédant ses propres institutions, syndicales, scolaires (Mériot, 2002), et ses propres dispositifs de jugement tels les associations de Chefs ou les guides gastronomiques reconnus par la profession (Karpik, 2000).

Les données sur lesquelles nous nous fondons sont issues d'une enquête de terrain réalisée (entre février et juillet 2006) par questionnaire en face à face, auprès d'environ trois cents restaurateurs de la métropole lilloise (dans le Nord de la France), de tous styles de cuisine et de toutes gammes de prix. Construit sur la base d'une pré-enquête qualitative (dix entretiens semi-directifs réalisés entre avril et octobre 2005), ce questionnaire comporte trois types de variables: premièrement, les attributs du restaurateur (âge, ancienneté, expérience, diplôme, récompenses, etc.) ; deuxièmement, les caractéristiques de son restaurant (taille, style de cuisine, prix pratiqués, nombre de salariés, type et nombre de fournisseurs etc.) ; troisièmement, les variables de réseau. Par l'intermédiaire d'un questionnaire sociométrique, nous enregistrons les liens que chaque restaurateur entretient avec les autres restaurateurs du marché. D'où l'importance de l'ancrage territorial et géographique de notre marché et de notre enquête. Par le générateur de nom suivant: "quels sont les restaurateurs que vous connaissez personnellement et qui vous connaissent personnellement aussi évidemment? », chaque 
restaurateur est amené à dresser la liste complète de ses contacts personnels dans le milieu. La formulation de cette question fait délibérément appel à la notion de réciprocité de sorte qu'il ne s'agit pas, pour les restaurateurs enquêtés, de citer des personnes « connues» (au sens médiatique du terme) mais bien des personnes avec lesquelles ils sont susceptibles d'être en lien. Une fois cette liste de contacts établie, la suite du questionnaire vise à identifier le type de lien entretenu avec chaque personne citée : ancienneté de l'interconnaissance, liens familiaux ou amicaux, et bien sûr les relations sociales, notamment le fait de discuter de la conjoncture entre restaurateurs, relation dont notre pré-enquête par entretiens nous a révélé l'importance :

Quand je vais au restaurant, j'ai tendance à discuter facilement avec les propriétaires en général. Quand je vais payer ben je dis : je suis restaurateur aussi et puis en général la conversation s'enclenche assez facilement. Je préfère avoir le maximum de relations dans le secteur quoi. Au moins on peut juger un peu la concurrence aussi quoi. (Restaurateur; cuisine traditionnelle régionale; menus à $12-20 € ; 50$ couverts)

Cet extrait d'entretien exploratoire traduit bien la démarche, postulée par White dans sa théorie du marché comme interface, à savoir celle où « les producteurs s'observent entre eux ». "Observer » les autres est ici considéré comme un moyen mis en œuvre par les entrepreneurs, collectivement engagés sur un marché, pour tenter de réduire l'incertitude inhérente à toute activité économique (Dallery, Eloire, Melmiès, 2010). Il ressort ainsi des entretiens l'importance quotidienne des "discussions de conjoncture " informelles entre les restaurateurs et ce, quel que soit leur style de cuisine ou leur gamme de prix :

On est tous dans la même barque, on est tous dans la même galère finalement, la restauration... on est tous confrontés aux mêmes problèmes heu... on est très curieux de savoir ce qui s'passe chez l'un chez l'autre, chez nous si on n'a pas marché on va s'inquiéter, on va s'demander : tiens, on a fait zéro couverts, est-ce que l'XX c'est pareil ? Donc on leur passe un petit coup de téléphone, si on les rencontre au $\mathrm{MIN}^{12}$, on se pose la question : tiens t'as fait du monde toi hier soir ? Et puis voilà, je pense, comme ça, à discuter et puis heu tout se joue là quoi. (Restaurateur; cuisine gastronomique ; menus à $32-64 € ; 80$ couverts)

Ou encore :

On parle de nos chiffres d'affaires, de la conjoncture, des salaires, de tout ça... (...) comme moi je suis au syndicat, j'en vois d'autres. (...). Non là où on échange beaucoup, c'est de savoir si on a fait du monde, pas de monde, ça c'est rigolo, ça c'est toujours le souci des restaurateurs. (Restaurateur; cuisine gastronomique; menus à $15-45 € ; 70$ couverts)

À partir des données d'enquête que nous avons recueillies, de manière systématique, au moyen du questionnaire, nous sommes en mesure de reconstituer le réseau des discussions informelles entre les restaurateurs lillois. Ce réseau peut être matérialisé sous la forme d'un graphe ( $c f$. figure 1) où les points sont les restaurateurs et les flèches les liens de discussion (qui discute avec qui ?). Ce réseau se présente sous la forme d'une seule et même composante, avec un centre, où les relations entre restaurateurs sont denses, et une périphérie où celles-ci s'effilochent et où certains sont même isolés. Comptant environ 2800 liens pour une densité de $3,4 \%^{13}$ et un taux de réciprocité de $58 \%$ ${ }^{14}$, ce graphe est incompréhensible tel quel, mais il va servir de base à une série d'analyses mobilisant les outils de réseaux sociaux. Ces analyses vont nous permettre de mieux appréhender la problématique du lien entre leadership relationnel et performance économique. 


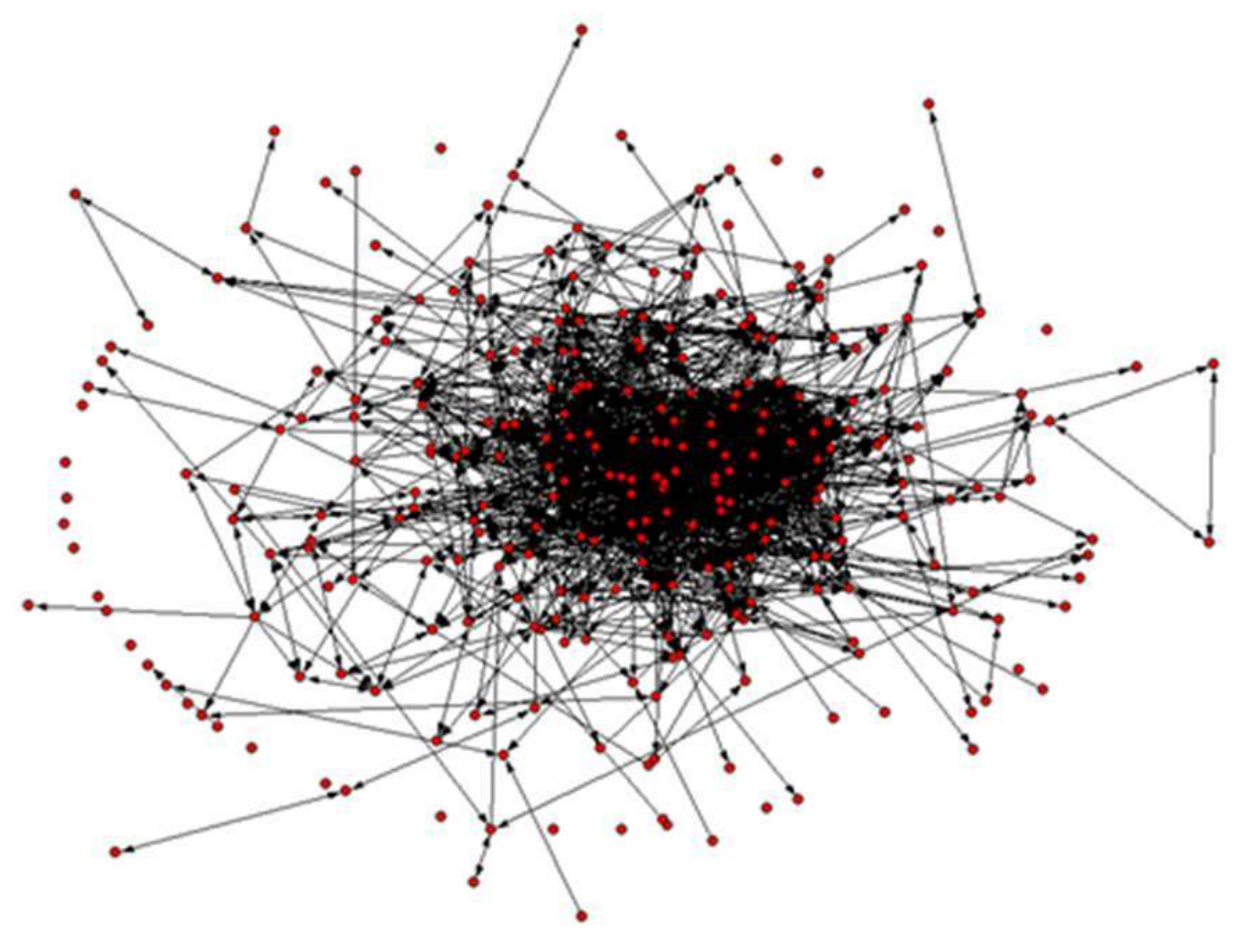

\subsection{La centralité permet-elle de repérer les leaders économiques ?}

29 À travers l'analyse de réseaux sociaux, la sociologie économique structurale dispose de différentes mesures "endogènes " du leadership: il s'agit des scores dits de centralité (Freeman, 1979; Wasserman, Faust, 1994), qui constituent différentes manières de calculer l'importance d'un acteur dans un réseau. Les mesures de centralité traduisent les positions relatives des acteurs au sein de la structure relationnelle, de sorte qu'« un acteur est d'autant plus central qu'il est engagé dans beaucoup de relations (directement ou indirectement). (...) Plus un acteur est central, plus il est actif dans le système » (Lazega, 1998, p. 43). Ces mesures de type quantitatif nécessitent évidemment des interprétations qualitatives, car il n'est pas certain qu'elles revêtent le même sens selon le milieu social ou le domaine d'activité étudié. Dans le cas des restaurateurs lillois, nous nous intéressons plus particulièrement à l'indicateur appelé indegree, qui recense le nombre de fois qu'un individu du réseau est choisi par les autres membres de ce réseau. Cet indicateur nous est apparu intéressant dans le cadre des discussions de conjoncture où l'objectif nous constatons que la distribution de cette mesure individuelle est très inégale comme le montre la figure 2 : 
Figure 3. Une distribution très inégale du score d'indegree

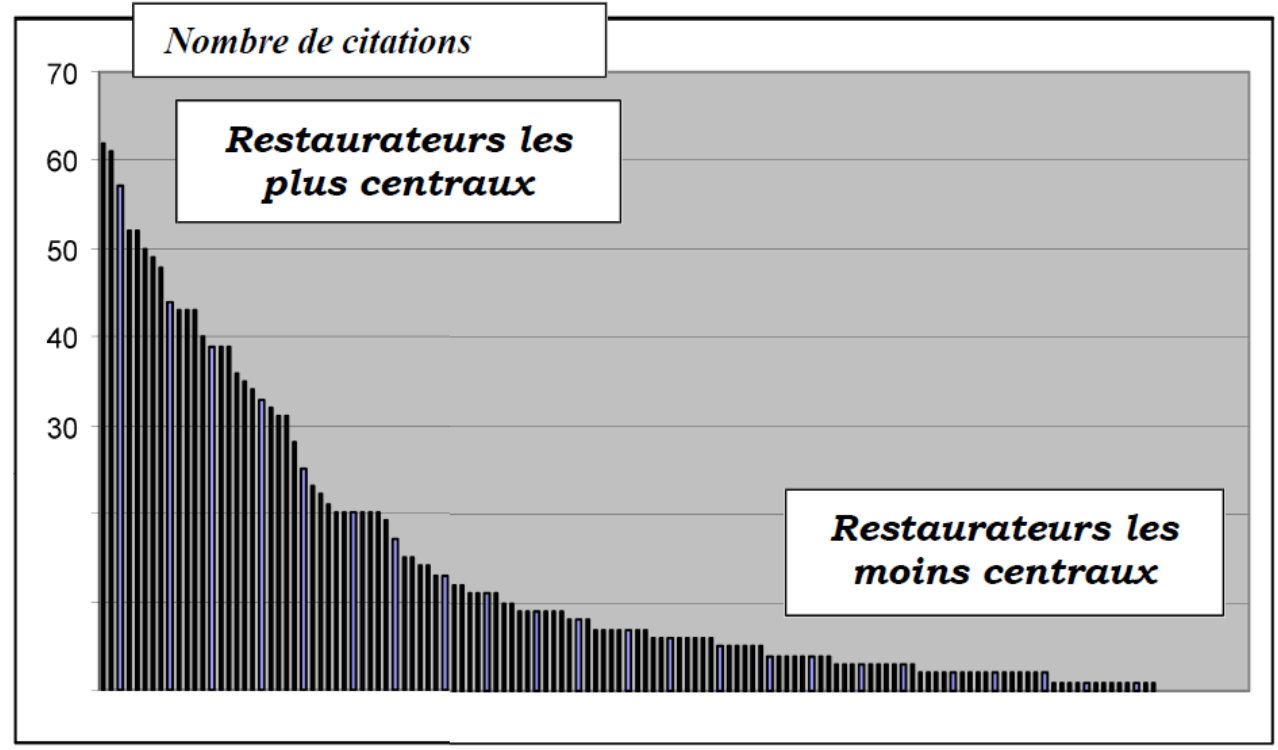

Certains restaurateurs sont très reconnus, au sens de très cités par leurs pairs (plus de 60 citations), quand d'autres restaurateurs ne sont cités par personne du milieu. Si on se réfère au graphe global des relations (figure 1), les restaurateurs les plus cités sont situés au cœur du réseau, quand les restaurateurs les moins cités sont situés en périphérie, avec peu ou pas de lien avec les autres restaurateurs du marché. En passant d'un indicateur de centralité relationnelle à un indicateur de performance économique, on constate la même distribution très inégale pour ce qui est du taux de marge nette ${ }^{15}$ :

Figure 4. Une distribution très inégale du profit

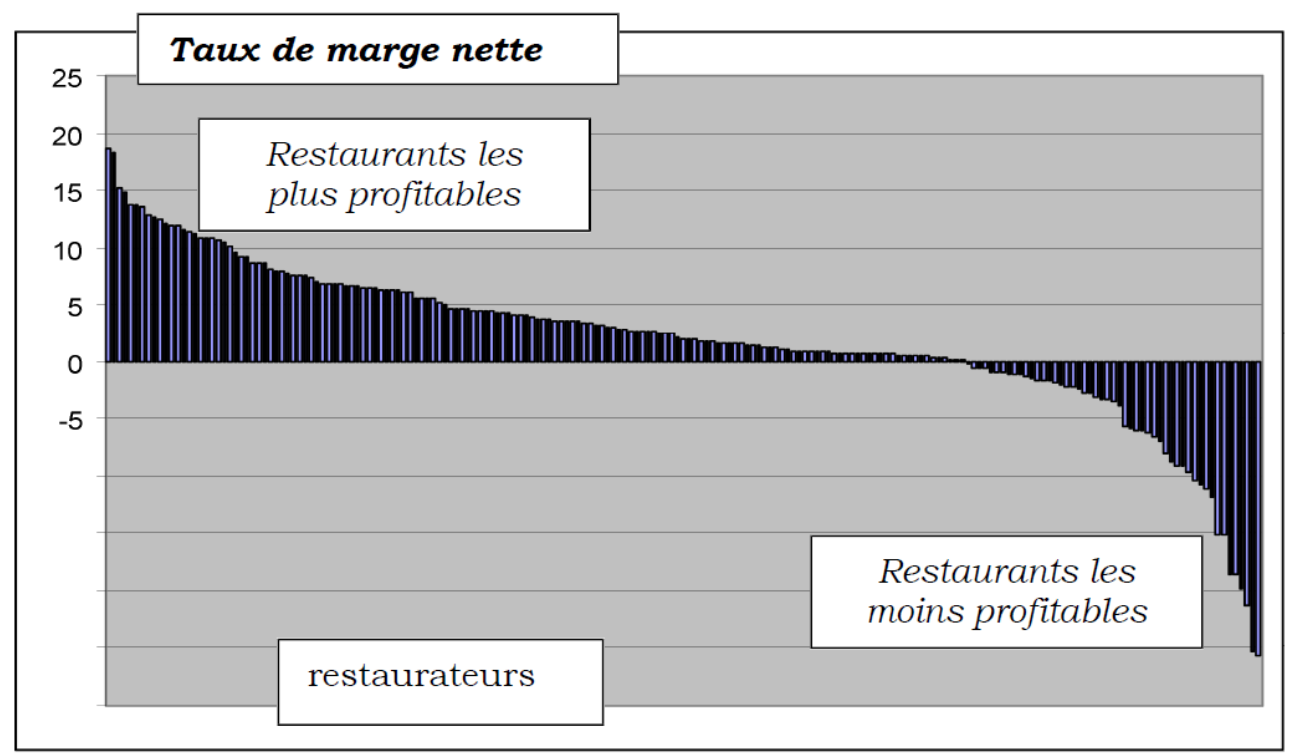

31 À partir de ces deux variables, dans un premier temps, l'hypothèse simpliste que nous formulons est la suivante : afin d'apporter un fondement empirique à la théorie du price leadership, nous postulons que les leaders économiques sur le marché sont ceux qui sont les plus regardés, donc qu'ils correspondent aux leaders relationnels au sein du réseau. 
Ainsi, selon la théorie du price leadership, on pourrait s'attendre à une corrélation significative et positive entre les deux variables d'indegree et de marge nette: selon la théorie, la firme leader fixe son prix avec une marge satisfaisante et des coûts au plus bas, et les firmes suiveuses doivent alors adopter ce prix, mais cela risque d'impliquer de devoir rogner sur leur marge pour y parvenir, leurs coûts étant a priori plus élevés que ceux des leaders. Pour résumer ici, sont leaders ceux qui sont regardés, et donc plus on est regardé, plus on devrait avoir une marge élevée.

Cette corrélation (tableau 1) n'est statistiquement pas significative : la première version, simpliste, de l'hypothèse de la théorie du price leadership n'est donc pas vérifiée. Au niveau du marché dans son ensemble, il ne semble pas exister de corrélation entre la centralité relationnelle et la performance économique.

Tableau 1. Analyse de corrélation entre indegree et marge nette

\begin{tabular}{|l|l|}
\hline & Marge nette \\
\hline Indegree & $-0.10(0.15)$ \\
\hline
\end{tabular}

Pour expliquer ce résultat, il convient de tenir compte d'un élément essentiel : la qualité. $\mathrm{Au}$ sein du marché de la restauration, nous avons repéré six stratégies de profit: la stratégie basée sur la qualité ${ }^{16}$ (choisie par $19 \%$ des établissements de notre population), celle basée sur le volume ${ }^{17}(16 \%)$, celle basée sur l'innovation (13\%), celle basée sur la combinaison qualité-volume $(10 \%)$, celle basée sur la spécialisation ${ }^{18}(9 \%)$ et celle basée sur la proximité (33\%).

Nous avons montré (Dallery, Eloire, Melmiès, 2009) que ces stratégies possédaient des rendements économiques différents ( $c f$. le tableau 2), de sorte que les restaurants qui adoptent les stratégies volume et spécialisation possèdent des rentabilités moyennes ( $4,04 \%$ pour le volume et $4,41 \%$ pour la spécialisation) qui apparaissent nettement supérieures à la moyenne de l'ensemble des stratégies (2,05\%). Et à l'inverse, que les restaurants qui adoptent une stratégie basée sur la qualité ou l'innovation ont une rentabilité moyenne nettement inférieure à la moyenne globale, au point que cette rentabilité moyenne s'avère même négative ${ }^{19}(-1,44 \%$ pour la qualité et $-0,79 \%$ pour l'innovation). Seules les entreprises capables de mêler la qualité au volume présentent des résultats économiques encourageants (2,82\%).

Tableau 2. Rentabilités moyennes des restaurants par stratégie de profit

\begin{tabular}{|l|l|l|l|l|}
\hline & $\begin{array}{l}\text { Moyenne des } \\
\text { taux de marge } \\
\text { nette }\end{array}$ & $\begin{array}{l}\text { Ecart-type des } \\
\text { taux de marge } \\
\text { nette }\end{array}$ & $\begin{array}{l}\text { Proportion } \\
\text { d'établissements à marge } \\
\text { nette négative }\end{array}$ & $\begin{array}{l}\text { Nombre de } \\
\text { données }\end{array}$ \\
\hline $\begin{array}{l}\text { Innovation- } \\
\text { Spécialisation }\end{array}$ & $-0,79 \%$ & $\mathbf{0 , 0 8 9}$ & $\mathbf{3 5 , 7} \%$ & $28(68 \%)$ \\
\hline Proximité & $\mathbf{+ 3 , 4 8 \%}$ & 0,063 & $18,4 \%$ & $49(47 \%)$ \\
\hline Qualité & $-1,44 \%$ & $\mathbf{0 , 0 7 5}$ & $\mathbf{4 5 , 2} \%$ & $42(72 \%)$ \\
\hline
\end{tabular}




\begin{tabular}{|l|l|l|l|l|}
\hline Qualité-Volume & $+2,82 \%$ & 0,056 & $18,5 \%$ & $27(87 \%)$ \\
\hline Spécialisation & $\mathbf{+ 4 , 4 1} \%$ & 0,046 & $11,7 \%$ & $17(59 \%)$ \\
\hline Volume & $+\mathbf{4 , 0 4} \%$ & 0,057 & $18,4 \%$ & $37(74 \%)$ \\
\hline Total & $+2,05 \%$ & 0,069 & $25,9 \%$ & $200(64 \%$ \\
\hline
\end{tabular}

De tels constats expliquent pourquoi notre analyse du lien « indegree - marge nette », ne prenant pas en compte la question de la qualité, ne peut aboutir à un résultat satisfaisant. $\mathrm{Ce}$, d'autant plus que, comme nous l'avons aussi montré ailleurs (Eloire, 2010), l'indegree est bien corrélé significativement et positivement à une autre variable de notre base de données : la « note de qualité » des restaurants (tableau 3).

Tableau 3. Analyse de corrélation entre indegree et marge nette

\begin{tabular}{|l|l|}
\hline & Note de qualité \\
\hline Indegree & $0.50(<.0001)$ \\
\hline
\end{tabular}

La note de qualité est une mesure objective du capital symbolique d'un établissement gastronomique. Nous avons construit cet indicateur, pour chaque établissement, à partir du relevé des citations et récompenses obtenues par eux dans les cinq principaux guides gastronomiques nationaux..$^{20}$ Cette note de qualité ne concerne qu'une cinquantaine d'établissements de notre population, soit environ $17 \%{ }^{21}$. Dans ces guides, nous constatons une relative homogénéité des jugements émis par les critiques, de sorte que la moitié, soit vingt-cinq établissements sont cités ou récompensés simultanément dans au moins trois guides différents, ce qui dénote une véritable concentration du capital gastronomique, qui est possédé par un petit nombre de restaurateurs. C'est, en fait, ce petit nombre de restaurateurs qui est le plus cité (plus hauts scores d'indegree), et donc le plus « observé » par les autres restaurateurs du réseau. Et comme la qualité n'est pas une stratégie rentable économiquement, cela renforce l'impossibilité d'un lien entre leadership relationnel et performance économique.

Qu'en est-il alors de la théorie du price leadership ? Doit-on en conclure que la théorie n'est pas vérifiée empiriquement sur notre marché, ou doit-on en conclure que l'analyse de réseaux sociaux, contrairement à ce que nous pensions, ne constitue pas un outil utile pour le repérage des leaders économiques (la théorie du price leadership resterait valide mais il y aurait une divergence entre leadership relationnel et leadership économique) ?

Avant de répondre définitivement à cette question, nous allons préciser notre analyse et descendre du niveau macro auquel nous l'avons pour l'instant placée, vers le niveau méso. En effet, nous souhaitons à présent introduire, dans notre analyse, la question de la qualité, c'est-à-dire la question de la structuration du marché et de la concurrence. Nous faisons donc l'hypothèse supplémentaire que, sur un marché, les firmes n'« observent " pas n'importe qui. Pour trouver les leaders économiques grâce aux relations, l'étude du contexte relationnel est donc incontournable : il ne faut pas se contenter d'une mesure 
brute de la centralité globale, mais prendre une mesure de centralité localisée, contextualisée. Pour ce faire, nous allons recourir à une analyse en termes de blockmodel.

\subsection{Le blockmodel permet-il de repérer les leaders économiques ?} permet de simplifier un réseau tel que celui de la figure 2. Il est fondé sur le principe de l'équivalence structurale (Lorrain, White, 1971) selon lequel, dans un réseau, deux individus sont interchangeables dès lors qu'ils ont les mêmes relations avec les mêmes individus. Le blockmodel s'applique à une matrice $(1,0)^{22}$, qu'il soumet à des algorithmes mathématiques afin de repérer les acteurs relativement structuralement équivalents ${ }^{23}$. Cette procédure aboutit à la constitution de blocks d'individus occupant ensemble, au sein du réseau, une position relativement similaire parce qu'ils ont à peu près les mêmes relations avec les mêmes individus. Ces blocks, qui peuvent être reliés entre eux, forment une structure relationnelle dans laquelle des groupes d'individus se forment, qui sont fondés, non pas comme c'est généralement le cas en sociologie, sur le partage d'attributs similaires (même sexe, même âge, etc.), mais sur le fait d'avoir des liens relativement similaires. ${ }^{24}$

Cet outil est particulièrement intéressant pour repérer, au sein d'un réseau, l'existence de sous-groupes non immédiatement décelables du fait de la complexité des graphes d'origine, tel celui de la figure 2. En appliquant une analyse en termes de blockmodel au réseau de discussions entre restaurateurs, notre objectif est de faire apparaître une structure relationnelle, composée de blocks, ou sous-groupes, de restaurateurs, dont nous faisons l'hypothèse qu'ils sont susceptibles de constituer les véritables lieux du leadership. Le blockmodel permet, en effet, de construire de nouveaux indicateurs de centralité, internes aux différents blocks, et conduisant à des mesures du leadership contextualisées par la structure relationnelle elle-même. Lorsque nous appliquons l'analyse en termes de blockmodel au réseau de discussions formé par les restaurateurs, nous obtenons une structure en huit blocks (cf. figure 4), où les points représentent des blocks, c'est-à-dire des sous-groupes de restaurateurs, et les flèches, des relations inter-blocks. 


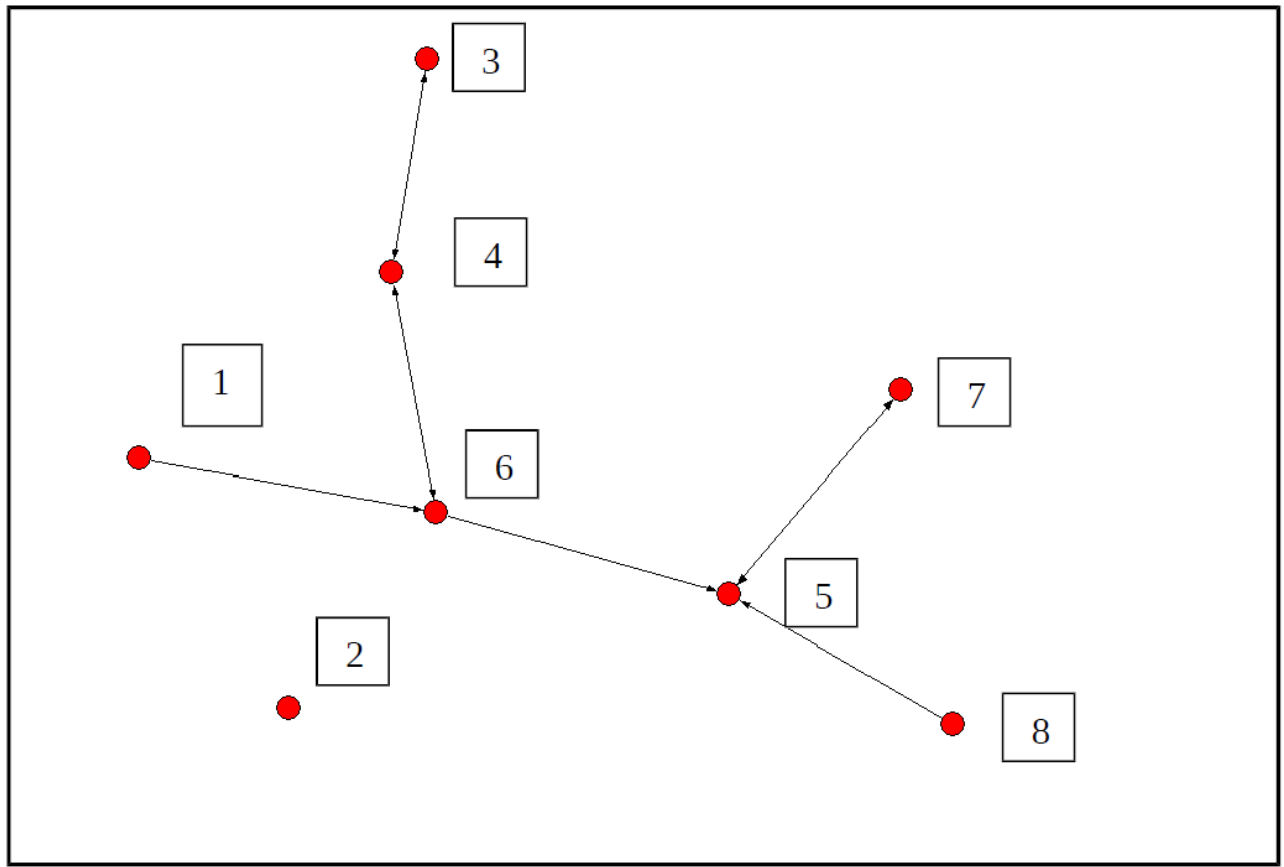

41 Comme l'indique le tableau 4 , le block le plus peuplé est le $n^{\circ} 1$ (64 restaurateurs), c'est aussi le moins dense $(3,7 \%)$ et celui où l'indegree moyen d'un restaurateur est le plus faible (2,7 relations par restaurateur en moyenne). A l'inverse, le block le moins peuplé est le $n^{\circ} 8$ (18). La particularité du block $n^{\circ} 2$ est qu'il est composé uniquement de restaurateurs qui sont isolés relationnellement, c'est-à-dire qui n'ont aucune relation ni entre eux ni avec personne au sein du réseau (d'où la densité nulle). Entre les blocks $\mathrm{n}^{\circ} 3$ et 4 , la relation est réciproque : les restaurateurs du block $\mathrm{n}^{\circ} 3$ ont tendance à citer les restaurateurs du block $n^{\circ} 4$, et inversement; entre les blocks $n^{\circ} 8$ et 5 , le lien est unilatéral : les restaurateurs du block $\mathrm{n}^{\circ} 8$ ont tendance à se réclamer d'une relation avec les restaurateurs du block $\mathrm{n}^{\circ} 5$, mais ces derniers ne les citent pas en retour, ce qui permet de repérer une relation de prestige asymétrique ${ }^{25}$. Les restaurateurs du block $\mathrm{n}^{\circ} 5$ ont en commun d'être plus recherchés par les membres des blocks $n^{\circ} 6,7$ et 8 . Les blocks $n^{\circ} 5$ et 6 sont la colonne vertébrale de la structure, ce sont aussi les blocks les plus denses (avec respectivement 29,9 et 23,1\%) et ceux dont l'indegree moyen est le plus élevé (27,6 et 16). Il convient, à présent, de compléter cette description formelle en examinant les attributs économiques et sociologiques des restaurateurs qui composent chacun des blocks.

Tableau 4. Caractéristiques des blocks

\begin{tabular}{|l|l|l|l|l|}
\hline & Blocks & Effectifs & Densité & Indegree \\
\cline { 2 - 5 } & 1 & 64 & $3,7 \%$ & 2,4 \\
\cline { 2 - 6 } Blockmodel réseau de discussions & 2 & 22 & $0 \%$ & 0 \\
\cline { 2 - 6 } & 3 & 45 & $8,2 \%$ & 7 \\
\cline { 2 - 6 } & 4 & 42 & $8,4 \%$ & 9,1 \\
\hline
\end{tabular}


Les restaurateurs des blocks $n^{\circ} 1,2$ et 3 disposent d'un faible poids économique et gastronomique sur le marché : leur nombre de clients est plutôt faible voire très faible, de même que le chiffre d'affaires; ils ne possèdent pas de note de qualité; leur style de cuisine est plutôt brasserie, cuisine traditionnelle ou pizzeria. Les restaurateurs du block $\mathrm{n}$ - 4 se caractérisent, eux aussi, par un poids gastronomique faible, mais se différencient des trois précédents blocks par un poids économique plus élevé, que ce soit en termes de nombre de clients, ou de volume de chiffre d'affaires. Le profil économique et sociologique du block $\mathrm{n}^{\circ} 6$ est marqué par une certaine ambigüité : si le poids économique y est relativement élevé, la cuisine possède aussi une réelle orientation gastronomique. Ce block centralise une dose importante de capital économique mais aussi symbolique. Le block $\mathrm{n}^{\circ} 5$ est composé de restaurateurs disposant d'un poids économique relativement élevé, mais surtout d'un poids gastronomique important : les prix pratiqués sont élevés, une note de qualité est généralement possédée, le chef d'entreprise y est aussi Chef cuisinier, et possède un diplôme en hôtellerie-restauration, son expérience professionnelle est solide et prestigieuse. Ce qui caractérise les membres du block $\mathrm{n}^{\circ} 7$, c'est moins leur poids économique ou gastronomique que leur diplôme et leur solide expérience professionnelle, leur fonction de cuisinier au sein de l'établissement et leur engagement dans la formation d'apprentis. Enfin, le block 8 se distingue par un faible poids économique et gastronomique sur le marché, et le partage plus fréquent d'attributs du type : travaille en couple, avec apprentis, et a de la famille dans le métier. Leurs établissements sont plutôt de petite taille, et la cuisine de style traditionnel.

Comme le montrent ces descriptions par les attributs, au sein des différents blocks, on est en mesure de faire ressortir des profils sociologiques de restaurateurs relativement cohérents. Elles constituent une autre illustration de la corrélation que nous avons mise au jour, plus haut, entre la note de qualité et l'indegree puisque les blocks $n^{\circ} 5$ et 6 , considérés comme les plus centraux, semblent rassembler majoritairement des restaurants de style gastronomique.

\section{Leadership collectif}

Une première analyse du lien entre réseau et leadership peut être envisagée au niveau collectif, c'est-à-dire non pas entre restaurateurs individuellement, mais entre blocks de restaurateurs ( $c f$. Tableau 5). L'objectif est toujours de tester si les leaders identifiés grâce à l'analyse des réseaux réalisent des marges de profit plus importantes que les autres, grâce à leurs capacités à imposer leur prix et à mieux contrôler leurs coûts :

Tableau 5. Rentabilité moyenne au sein des blocks

\begin{tabular}{|l|l|l|l|l|l|}
\hline $\begin{array}{l}\text { Blockmodel réseau de } \\
\text { discussions }\end{array}$ & Blocks & $\begin{array}{l}\text { Marge } \\
\text { nette }\end{array}$ & $\begin{array}{l}\text { Prix } \\
\text { moyen ? }\end{array}$ & Indegree & $\begin{array}{l}\text { Note de } \\
\text { qualité }\end{array}$ \\
\cline { 2 - 7 } & 1 & $+2,55 \%$ & $14,1(4,2)--$ & $1,2--$ & $0,2--$ \\
\cline { 2 - 7 } & 2 & $+6,59 \%$ & $12,5(3,1)--$ & $0--$ & $0--$ \\
\hline 3 & $+3,80 \%$ & $18(6,1)-$ & $2,4-$ & $0,2--$ \\
\hline & 4 & $+2,00 \%$ & $16,9(5,4) 0$ & $2,8-$ & $0,4--$ \\
\hline
\end{tabular}




\begin{tabular}{|l|l|l|l|l|}
\hline 5 & $+0,71 \%$ & $27,4(14,4)++$ & $6,7++$ & $10++$ \\
\hline 6 & $+4,31 \%$ & $24,1(17,3)+$ & $4,1++$ & $7,2++$ \\
\hline 7 & $+0,48 \%$ & $23(9,3)+$ & $3,7+$ & $2,6+$ \\
\hline 8 & $+3,67 \%$ & $18,1(5,5)+$ & $1+$ & $0,2+$ \\
\hline
\end{tabular}

\begin{tabular}{|l|l|l|l|}
\hline & Blocks & Leaders & Suiveurs \\
\cline { 2 - 4 } & 1 & 12 & 14 \\
\cline { 2 - 4 } Blockmodel Réseau de discussions & 2 & 12 & \\
\cline { 2 - 4 } & 4 & 20 & 16 \\
\cline { 2 - 5 } & 3 & 19 & 17 \\
\cline { 2 - 5 } & 5 & 35 & 20 \\
\cline { 2 - 5 } & 6 & 30,1 & 21 \\
\cline { 2 - 5 } & 7 & 28 & 19 \\
\cline { 2 - 5 } & 8 & 23 & 17 \\
\cline { 2 - 5 } & 8 & 23 & 12 \\
\hline
\end{tabular}

Le block qui ressort avec la meilleure performance économique est le block $\mathrm{n}^{\circ} 2 . \mathrm{Ce}$ résultat va a priori à l'encontre de la théorie du price leadership, puisque les restaurateurs de ce block n'ont rien de leaders, étant donné qu'ils sont isolés relationnellement, ils ne sont cités par personne. Rappelons cependant pour expliquer cette bonne performance économique que ce block est formé par de petits établissements et que c'est le block dans lequel il manque le plus de données de marges de profit. Autre explication possible à ce résultat: ces petits restaurants optent pour une cuisine simple, provenant de la grande distribution, les coûts y sont donc moindres, et une marge satisfaisante est plus facilement réalisable. Il s'agit ici d'une stratégie de report de contrainte sur la qualité : pour que le restaurateur survive et dégage une marge de profit suffisante, il doit vendre des produits standardisés, uniformisés au goût moyen (comme le sont les produits industriels achetés dans la grande distribution), au lieu de cuisiner, de transformer luimême des produits achetés auprès de petits fournisseurs. Ces restaurants offrent une cuisine bon marché, mais comparativement aux autres restaurateurs, ils baissent plus fortement la qualité (coûts) qu'ils ne baissent les prix, de sorte que leur marge de profit en ressort plus élevée.

Outre le cas particulier du block $\mathrm{n}^{\circ} 2$, le second block à obtenir une marge élevée est le block $\mathrm{n}^{\circ} 6$. Il s'agit cette fois d'un résultat beaucoup plus conforme à ce qu'on est en droit 
d'attendre avec la théorie du price leadership. Les restaurateurs de ce block forment le véritable leader collectif du marché :

i. dans la structure globale du blockmodel, ils sont relativement centraux et sont regardés par les restaurateurs du block $\mathrm{n}^{\circ} 1$ et du block $\mathrm{n}^{\circ} 4$ qui sont les blocks les plus peuplés;

ii. ils ont un indegree global élevé tout comme les restaurateurs des blocks $n^{\circ} 5$ et 7 , mais à la différence du block $\mathrm{n}^{\circ} 5$, leur indegree ne repose pas tant sur une reconnaissance interne au block (densité), que sur une reconnaissance qui puise aussi plus largement sa source chez des restaurateurs d'autres blocks. l'ensemble des restaurateurs du marché. Et conformément à la théorie du price leadership, portée cette fois à un niveau méso, le leader, ici collectif, structure le marché (production, coûts) et réalise les marges les plus élevées en bénéficiant de son pouvoir sur le marché. La qualité est présente, les coûts sont donc élevés, mais la reconnaissance par les acteurs du marché (les pairs, mais aussi les clients) permet à ces établissements de faire payer des prix élevés et de réaliser ainsi des marges de profit importantes.

Cableau 5 laisse aussi apparaitre un résultat, a priori paradoxal, quant au statut du block $\mathrm{n}^{\circ}$ 5. Compte tenu de sa position relativement centrale dans la structure relationnelle ( indegree global le plus élevé, position centrale dans le blockmodel à travers le sens des relations au sein du blockmodel), on pouvait s'attendre à ce que ce block réalise une belle performance économique. Or, la marge nette moyenne des restaurateurs de ce block est très faible. centralité relationnelle n'est pas à même d'identifier les leaders de marché ? Selon nous, ce résultat suggère davantage que le leadership relationnel n'a pas qu'une dimension économique. Si ces restaurateurs, qui semblent bénéficier d'une centralité relationnelle forte, n'ont pas une marge de profit élevée, c'est peut-être parce que la logique de leur leadership relationnel n'est pas économique (faire du profit), mais symbolique (faire de la qualité) ${ }^{26}$. Ces restaurateurs sont engagés dans une concurrence de statut (Lazega, Mounier, 2002 ; Lazega, 2009) pour l'accès à la reconnaissance des pairs, mais cette compétition ne paie pas sur le plan économique, elle n'est pas (suffisamment) reconnue par les clients : les coûts n'étant pas reconnus par les clients, le prix qu'ils acceptent de payer (ou le nombre de clients qui acceptent de payer le prix) sera inférieur à ce qui permettrait à ces restaurateurs de dégager une meilleure marge. La différence essentielle entre le block $\mathrm{n}^{\circ} 5$ et le block $\mathrm{n}^{\circ} 6$ réside donc dans le type de capital visé : les restaurateurs du block $\mathrm{n}^{\circ} 5$ visent une reconnaissance gastronomique, et leur leadership relationnel témoigne alors de cette quête de voir sa cuisine reconnue dans le milieu de la "haute cuisine", de se voir coopté parmi les grands; les restaurateurs du block $n^{\circ} 6$ visent un capital économique, un profit commercial, et leur leadership relationnel consacre la réussite d'une entreprise économique, la restauration étant un domaine d'activité comme un autre.

\section{Leadership individuel}

Une seconde analyse peut être effectuée au niveau de chaque block. Le principe est de repérer, au sein de chaque block, les restaurateurs qui sont les plus centraux, c'est-à-dire les leaders d'un point de vue relationnel. Nous distinguerons alors deux catégories différentes de restaurateurs, les leaders internes et les «suiveurs ». Afin de vérifier, à 
propos de la théorie du price leadership, notre hypothèse de départ sur le lien entre indegree et leadership économique, nous nous attendons à obtenir des résultats tels que les leaders relationnels, au sein de chaque block, c'est-à-dire les plus « observés », soient aussi les leaders économiques, c'est-à-dire les plus profitables, si on compare leur marge à celle des suiveurs.

Le repérage des leaders internes est réalisé de manière très empirique : nous nous fondons à la fois sur l'indicateur quantitatif des centralités internes, pour chaque restaurateur dans son block, et sur notre connaissance qualitative des données et du terrain (cf. en annexe les graphes représentant la configuration interne de chaque block avec ses leaders - en bleu - et ses suiveurs - en blanc). Comme le montre le tableau 6, le nombre de leaders diffère selon la taille et la configuration de chaque block. On passe ainsi de 21 leaders internes au sein du block ${ }^{\circ} 5$ à deux leaders internes au sein du block n ${ }^{\circ} 8$, et il n'y a aucun leader interne dans le block ${ }^{\circ} 2$ dans la mesure où il n'est composé que de restaurateurs isolés.

Tableau 6. Indegree et marge nette des leaders et des suiveurs block par block

\begin{tabular}{|l|l|l|l|l|l|l|l|l|}
\hline Leaders internes & Block 1 & Block 2 & Block 3 & Block 4 & Block 5 & Block 6 & Block 7 & Block 8 \\
\hline Effectif (marges disponibles) & $8(6)$ & & $11(10)$ & $9(8)$ & $21(18)$ & $8(7)$ & $11(6)$ & $2(0)$ \\
\hline Indegree global & 7 & & 14,54 & 21,11 & 44,86 & 31,7 & 37,18 & 6 \\
\hline Indegree interne au block & 4,62 & & 7,18 & 9,55 & 21,29 & 6,6 & 11,55 & 4 \\
\hline Taux de marge nette moyen & $+6,28 \%$ & & $+6,32 \%$ & $+3,58 \%$ & $-0,47 \%$ & $+5,84 \%$ & $+0,81 \%$ & - \\
\hline
\end{tabular}

\begin{tabular}{|l|l|l|l|l|l|l|l|l|}
\hline Suiveurs & Block 1 & Block 2 & Block 3 & Block 4 & Block 5 & Block 6 & Block 7 & Block 8 \\
\hline $\begin{array}{l}\text { Effectif } \\
\text { disponibles) }\end{array}$ & $61(35)$ & $24(8)$ & $39(25)$ & $41(27)$ & $27(17)$ & $16(11)$ & $17(9)$ & $16(8)$ \\
\hline Indegree global & 1,81 & 0 & 4,92 & 6,56 & 14,19 & 8 & 6,64 & 3 \\
\hline Indegree interne au block & 1,18 & 0 & 2,05 & 1,90 & 5,11 & 2,4 & 2 & 0,56 \\
\hline Taux de marge nette moyen & $+1,92 \%$ & $+6,59 \%$ & $+2,79 \%$ & $+1,53 \%$ & $+1,95 \%$ & $+3,35 \%$ & $+0,27 \%$ & $+3,67 \%$ \\
\hline
\end{tabular}

Ces tableaux permettent de comparer, block par block, d'un point de vue relationnel ( indegree) et économique (marge nette), la situation des leaders internes par rapport à celle des suiveurs. En ce qui concerne les aspects relationnels, d'abord, on constate premièrement que l'indegree global est bien plus élevé pour les leaders que pour les suiveurs. Deuxièmement, l'indegree interne, à partir duquel nous avons repéré les leaders internes, est évidemment, par construction, toujours supérieur chez les leaders, avec des écarts plus ou moins importants par rapport aux suiveurs. 
54 En ce qui concerne l'aspect économique, maintenant, la comparaison, block par block, des taux de marges nette, montre bien l'effet du leadership relationnel. L'hypothèse de départ et la théorie du price leadership semblent confirmées ici : les leaders relationnels semblent bien disposer d'un pouvoir sur le marché qui leur octroie une marge supérieure. Le seul block pour lequel notre hypothèse ne se vérifie pas est, à nouveau, le block $\mathrm{n}^{\circ} 5$. Pourquoi ? Parce que les restaurateurs de ce block ne sont pas pris dans une concurrence économique mais dans une concurrence de statut, dans une compétition " gastronomique ». Le fait d'être « observé » par les autres n'est pas alors l'indice d'un leadership économique permettant une meilleure marge de profit. La centralité est ici gage d'une reconnaissance de la qualité gastronomique de l'établissement. Le problème est alors que la gastronomie exige des coûts élevés avec le besoin d'avoir de nombreux fournisseurs de qualité, un faible poids des dépenses dans la grande distribution, etc. (cf. Tableau 7). De plus, la logique économique empêche de répercuter l'intégralité de ces coûts sur le prix de vente (ce qui finirait par faire fuir les clients). La logique de la compétition « gastronomique » est ici d'accepter une marge plus faible, le temps d'asseoir sa réputation, puis éventuellement de devenir leader parmi les leaders (et rejoindre le block $n^{\circ} 6$ ?) en réussissant à devenir leader gastronomique et leader économique (la réputation étant faite, il devient possible de répercuter les coûts de la qualité sur le prix sans sacrifier sa marge). Par ailleurs, on remarque également que la compétition gastronomique n'est pas absente des autres blocks, où les leaders ont aussi une tendance à avoir plus de fournisseurs et moins de dépenses dans la grande distribution, signes objectifs d'une meilleure qualité gastronomique.

Tableau 7. Comparaison block par block entre leaders et suiveurs en fonction de différents indicateurs de qualité gastronomique

\begin{tabular}{|c|c|c|c|c|c|c|c|c|}
\hline Leaders individuels & Block 1 & $\begin{array}{l}\text { Block } \\
2\end{array}$ & $\begin{array}{l}\text { Block } \\
3\end{array}$ & Block 4 & Block 5 & $\begin{array}{l}\text { Block } \\
6\end{array}$ & Block 7 & Block 8 \\
\hline Taux de marge nette moyen & $6,28 \%$ & & $6,32 \%$ & $3,58 \%$ & $-0,47 \%$ & $5,84 \%$ & $0,81 \%$ & - \\
\hline Note de qualité & 0 & & 0,64 & 0 & 20,84 & 15,87 & 6,55 & 0 \\
\hline Nombre de fournisseurs & 6,13 & & 9,36 & 10,11 & 10 & 14,5 & 7,36 & 3,5 \\
\hline $\begin{array}{l}\text { Part des dépenses dans la } \\
\text { grande distribution }^{27}\end{array}$ & $36,88 \%$ & & $9,18 \%$ & $15,77 \%$ & $25,19 \%$ & $9,37 \%$ & $51,82 \%$ & $75,00 \%$ \\
\hline
\end{tabular}

\begin{tabular}{|l|l|l|l|l|l|l|l|l|}
\hline Suiveurs & Block 1 & Block 2 & Block 3 & Block 4 & Block 5 & $\begin{array}{l}\text { Block } \\
6\end{array}$ & Block 7 & Block 8 \\
\hline Taux de marge nette moyen & $1,92 \%$ & $6,59 \%$ & $2,79 \%$ & $1,53 \%$ & $1,95 \%$ & $3,35 \%$ & $0,27 \%$ & $3,67 \%$ \\
\hline Note de qualité & 0,21 & 0 & 0,13 & 0,54 & 1,63 & 2,87 & 0,18 & 0,25 \\
\hline Nombre de fournisseurs & 5,75 & 4,08 & 7,10 & 7,41 & 7,33 & 8,25 & 6,24 & 6 \\
\hline
\end{tabular}




\begin{tabular}{|l|l|l|l|l|l|l|l|l|}
\hline $\begin{array}{l}\text { Part des dépenses dans la } \\
\text { grande distribution }\end{array}$ & $37,02 \%$ & $36,88 \%$ & $33,92 \%$ & $20,02 \%$ & $33,33 \%$ & $20,5 \%$ & $45,59 \%$ & $40,00 \%$ \\
\hline
\end{tabular}

\section{Conclusion} gastronomique.
Dans cet article, nous nous sommes intéressés à la théorie du price leadership, et nous avons entrepris de la tester en la confrontant à des données d'enquête. En amont de cette procédure de test, nous avons amendé la théorie elle-même en clarifiant la notion de leadership. Alors qu'auparavant, la définition du leader sur un marché renvoyait à des conceptions restrictives (cas de la seule concurrence imparfaite) et peu pertinentes (inadaptées à la réalité), nous avons opté pour une définition relationnelle du leadership. Ce premier apport de notre travail démontre les bénéfices à attendre de recherches pluridisciplinaires en sciences humaines, puisque c'est bien la sociologie des réseaux qui offre une définition convaincante du leadership pour l'économie post-keynésienne.

Munis de cette définition du leadership sur un marché, et à l'aide des outils de l'analyse des réseaux sociaux, nous avons ensuite entrepris de vérifier les prédictions de la théorie du price leadership : les leaders relationnels réalisent-ils des marges de profit supérieures? Nos données d'enquête offrent alors une réponse contrastée, puisque les leaders, que ce soit sur le plan individuel ou collectif, obtiennent, la plupart du temps, ces meilleures performances économiques. Lorsque le leadership relationnel ne se conjugue pas avec les meilleures performances économiques, deux explications peuvent être avancées : c'est d'une part pour le cas des restaurateurs isolés, parce qu'ils tirent vers le bas la qualité en se fournissant dans la grande distribution et en offrant des plats préparés industriellement; et d'autre part, pour les restaurateurs gastronomiques, parce qu'ils sont engagés dans une compétition de statut, une recherche de la reconnaissance

C'est là où l'apport de la sociologie est sans doute le plus intéressant, la définition relationnelle du leadership permet de mettre en évidence des « niches » où ce ne sont pas les logiques économiques qui gouvernent, mais où la centralité relationnelle révèle des espaces de compétition symbolique : au sein de ces niches, le leadership relationnel ne permet pas une meilleure performance économique, mais il est absolument nécessaire pour l'obtention d'une reconnaissance symbolique, la réussite économique étant un objectif secondaire, souvent difficile à atteindre (Dallery, Éloire et Melmiès, 2009). Le leadership relationnel traduit donc un leadership économique ou symbolique selon les cas.

$\mathrm{Au}$ bout du compte, la confrontation entre économie post-keynésienne et sociologie économique structurale modifie la vision classique du marché. Il n'y a pas de théorie systématique, et il est toujours nécessaire de procéder à une contextualisation, à l'étude des caractéristiques sociologiques des acteurs.

Les résultats empiriques obtenus ici, sur la base d'une théorie du leadership que nous pensons solide, ont vocation à être testés à plus large échelle et dans d'autres secteurs. 


\section{BIBLIOGRAPHIE}

Bourdieu Pierre, 2000, Les structures sociales de l'économie, Paris, Seuil.

Dallery Thomas, 2010, Le divorce rentabilité / croissance dans le capitalisme financiarisé. Changements de régimes, équilibres, instabilités et conflits, Thèse de doctorat, Université Lille 1.

Dallery Thomas, van Treeck Till, 2010, «Conflicting Claims and Equilibrium Macroeconomic Adjustment Processes in a Stock-Flow Consistent Macromodel », Review of Political Economy, forthcoming.

Dallery Thomas, Eloire Fabien, Melmiès Jordan, 2009, « La fixation des prix en situation d'incertitude et de concurrence : Keynes et White à la même table », Revue Française de Socioéconomie, $2^{\mathrm{e}}$ semestre, $\mathrm{n}^{\circ}$ 4, La Découverte, Paris, p. 177-198.

Dallery Thomas, Eloire Fabien, Melmiès Jordan, 2010, « Confrontation entre théorie postkeynésienne et sociologie économique structurale », Innovations, Cahiers d'Economie de l'Innovation, L'Harmattan, $1^{\text {er }}$ trimestre, $n^{\circ} 31$.

Eichner Alfred, 1976, The Megacorp and Oligopoly, Armonk, New-York, M.E. Sharpe.

Eloire Fabien, 2010, « Une approche sociologique de la concurrence sur un marché. Le cas des restaurateurs lillois », Revue Française de Sociologie, $\mathrm{n}^{\circ} 3$.

Freeman, Linton C., 1979, «Centrality in social networks: conceptual clarification », Social networks, $\mathrm{n}^{\circ} 1$, pp. 215-239.

Gadrey Jean. et al., 2002, Hôtellerie-restauration : héberger et restaurer l'emploi, les cas français, américain et japonais, La Documentation Française, Paris.

Kalecki Michal, 1954, Theory of Economic Dynamics, George Allen and Unwin, Londres.

Karpik Lucien, 2000, « Le Guide Rouge Michelin », Sociologie du Travail, 42, pp. 369-389.

Karpik Lucien, 2007, L'économie des singularités, NRF, Gallimard.

Kenyon Peter, Harcourt Geoff, 1976, «Pricing and the investment decisions », Kyklos, n³, pp. 33-50.

Lavoie Marc, 2004, L'économie postkeynésienne, Éditions La Découverte, coll. Repères.

Lazega Emmanuel, 1998 [2007], Réseaux sociaux et structures relationnelles, Que sais-je, PUF.

Lazega Emmanuel, Mounier Lise, 2002, « Interdependent entrepreneurs and the social discipline of their cooperation: The research program of structural economic sociology for a society of organizations ", in Favereau, Lazega (eds), Conventions and Structures in Economic Organization: Markets, Networks, and Hierarchies, Cheltenham, Edward Elgar Publishing, pp. 147-199.

Lazega Emmanuel, 2009, « Théorie de la coopération entre concurrents : organisations, marchés et réseaux ", in. Steiner et Vatin, Traité de sociologie économique, PUF.

Lee Frederic, 2010, Post Keynesian Microeconomic Theory, Routledge, forthcoming.

Lorrain François, White Harrison C., 1971, « Structural equivalence of individuals in social networks ", Journal of Mathematical Sociology, $n^{\circ}$ 1, pp. 49-80. 
Melmiès Jordan, 2009, « Une lecture de l'histoire de la théorie des prix chez les post-keynésiens », Documents de Travail du Clersé, $\mathrm{n}^{\circ} 3$.

Melmiès Jordan, 2011, Prix, marges et accumulation : une théorie dynamique de la concurrence, Thèse de doctorat en sciences économiques, Université Lille1.

Mériot Sylvie-Anne, 2002, Le cuisinier nostalgique, entre restaurant et cantine, Paris, CNRS Editions. Robinson Joan, 1933 [1969], The Economics of Imperfect Competition, Palgrave Mac Millan, Londres. Wasserman Stanley, Faust, K., 1994, Social network analysis: methods and applications, Cambridge University Press.

White Harrison C., Boorman Scott, Breiger Ronald, 1976, « Social structure from multiple networks I. blockmodels of roles and positions ", American Journal of Sociology, n 81, pp. 730-870.

White Harrison C., 1981, « Where do markets come from? », American Journal of Sociology, 87 (3), pp. 517-587.

White Harrison C., 2002, Markets from networks. Socioeconomic models of production, Princeton, Princeton University Press.

Wood Adrian, 1975, A Theory of Profits, Cambridge, Cambridge University Press.

\section{ANNEXES}

\section{Block 1}

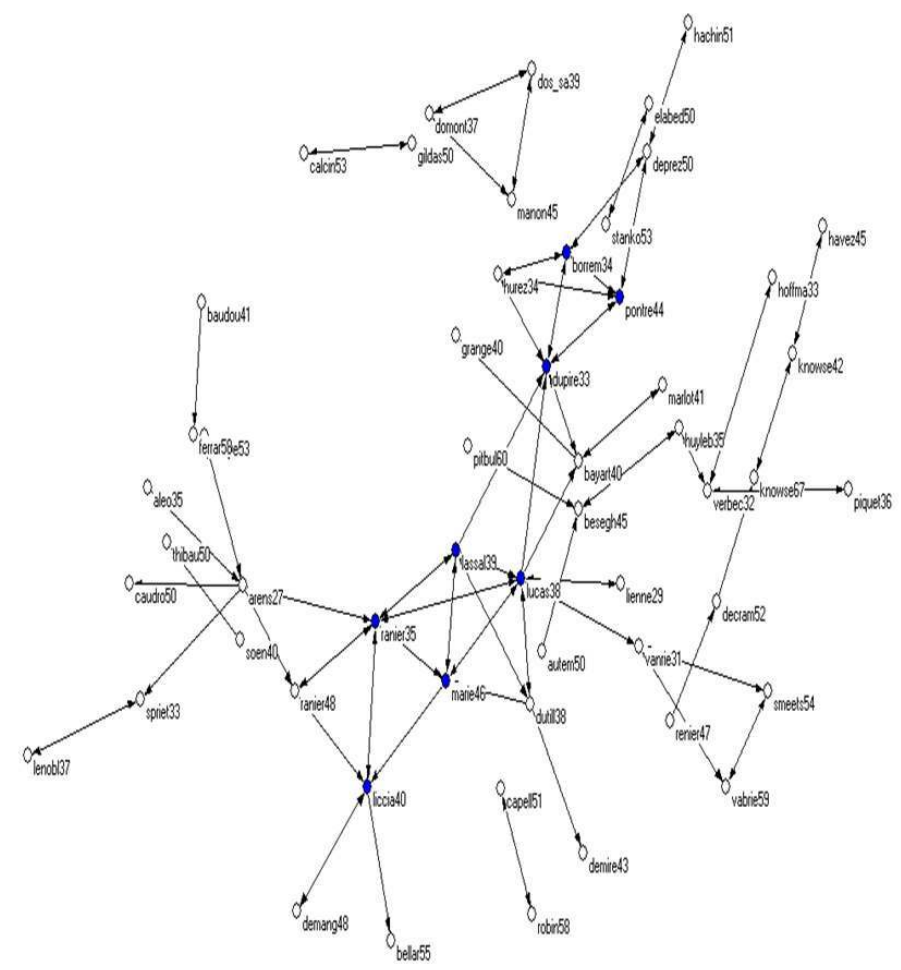


Block 3

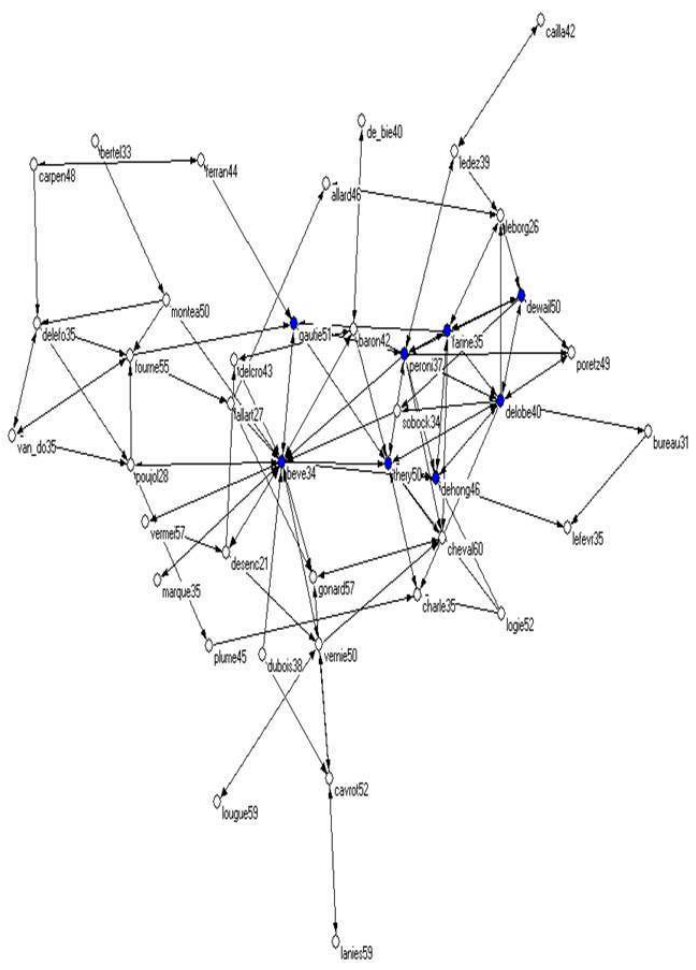

Block 4

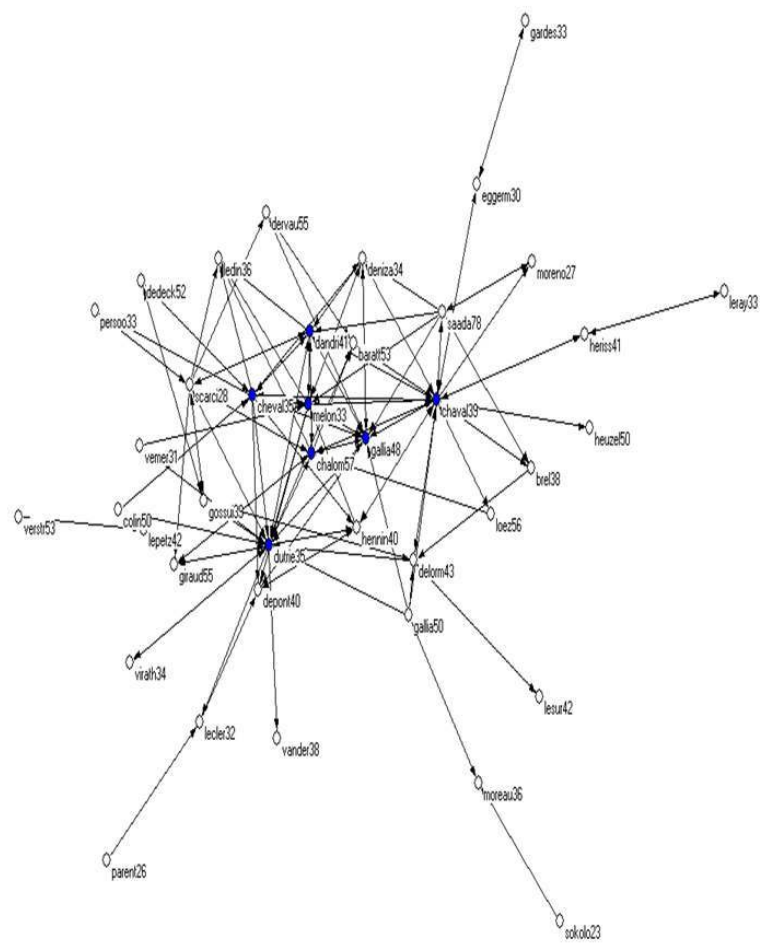


Block 5

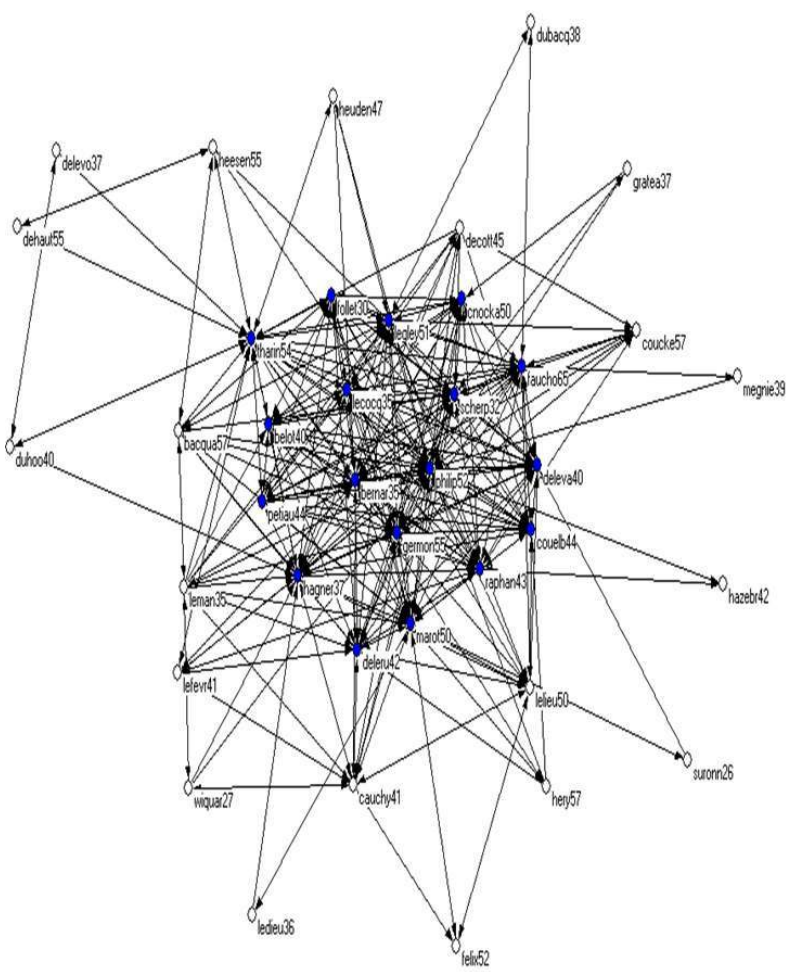

Block 6

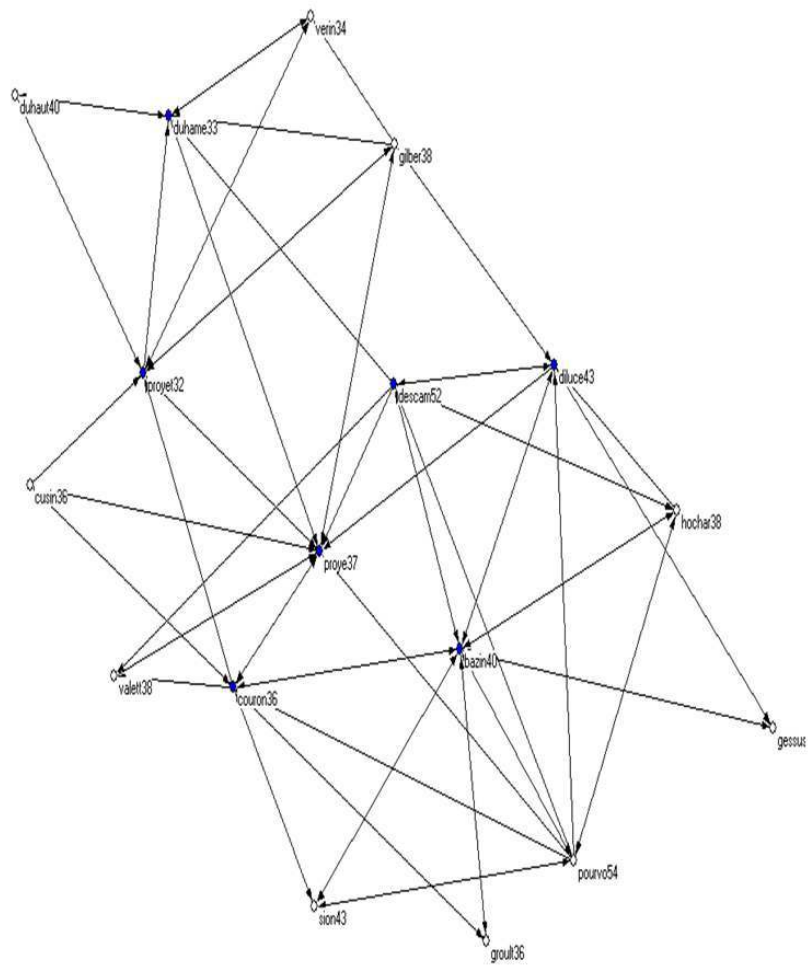


Block 7

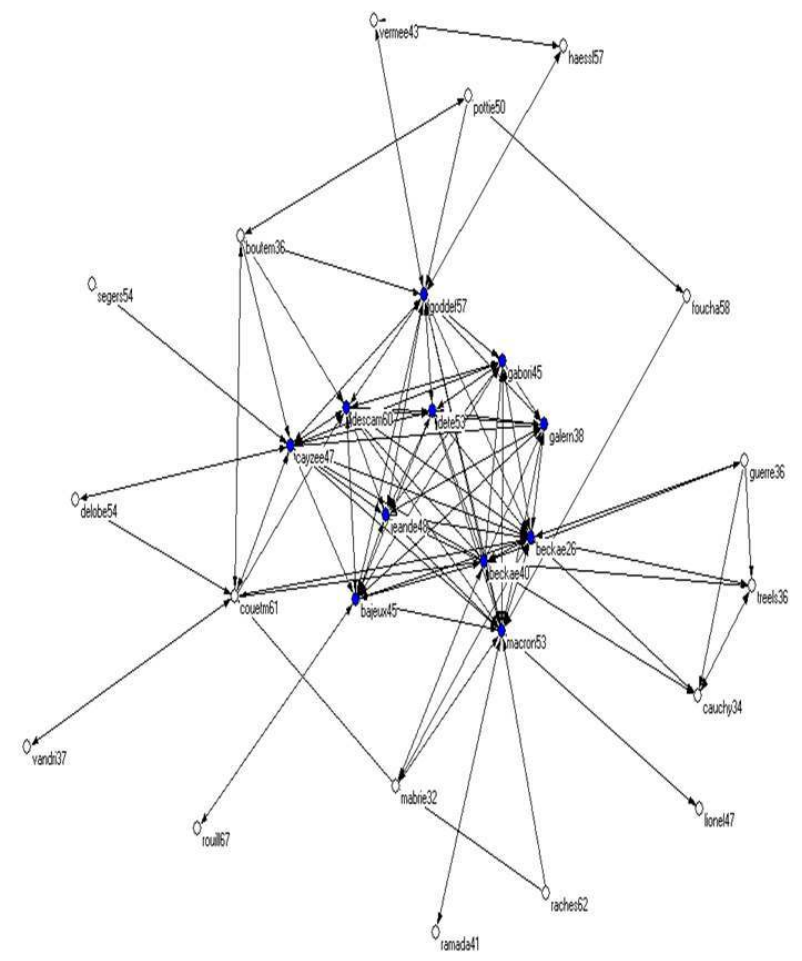

Block 8
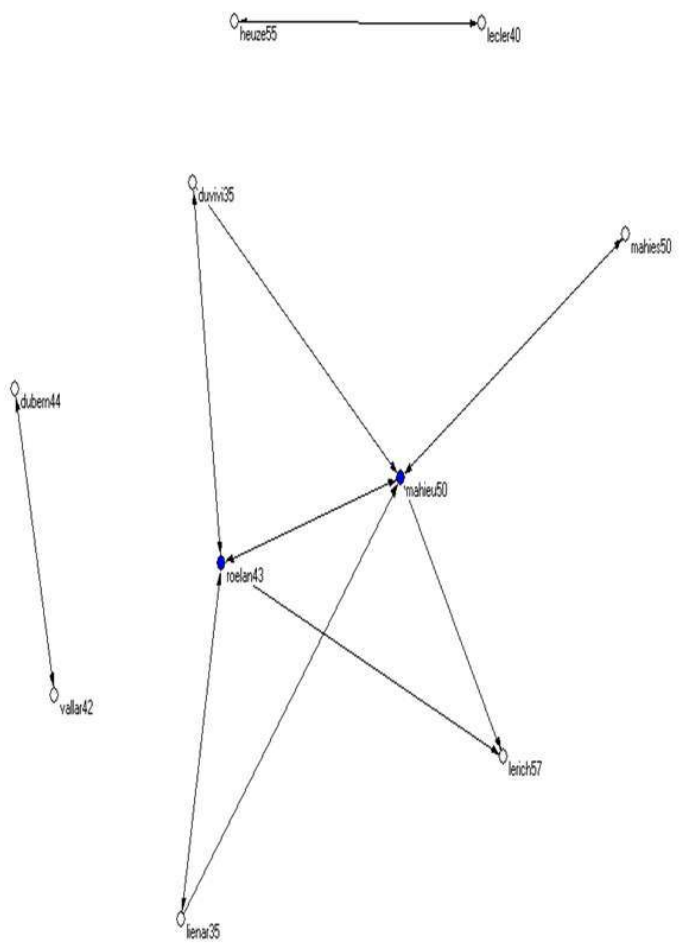


\section{NOTES}

1. Nous verrons plus loin dans l'article qu'une solution serait celle d'un report de contrainte sur la qualité ou sur les fournisseurs.

2. Pour illustrer notre propos, nous nous appuyons, tout au long de cet article, sur les données d'une enquête de terrain réalisée auprès de trois cents restaurateurs de la métropole lilloise (cf. la deuxième partie).

3. Pour des firmes déjà installées, ces questions ne se posent que dans une perspective dynamique de modification des réponses apportées par le passé.

4. Il existe différentes procédures de fixation des prix chez les Post-Keynésiens : le mark-up, le full cost, le target return pricing (Lavoie, 2004, p. 44 et suivantes). Mais toutes reposent sur l'ajout d'une marge à une mesure des coûts. Ces procédures ne diffèrent pas sur le principe, mais sur la manière de calculer la marge et/ou sur les coûts à prendre en compte.

5. Ce scenario renvoie à ce que Gadrey et al. (2002) nomment, à l'échelle macroéconomique, le modèle "à l'américaine », fondé sur le salariat, la franchise, et les chaînes, et caractérisé par « un assez grand nombre de mauvais emplois, peu payés, à fort turnover» (p. 20).

6. Cette hypothèse a été exposée en détail chez Alfred Eichner (1976) qui l'applique aux « megacorps ", grandes unités agissant dans un univers oligopolistique avec une hiérarchie leader/ suiveur entre firmes. Elle sera reprise et développée de nombreuses fois, par Adrian Wood (1975) ou Kenyon et Harcourt (1976) notamment.

7. On suppose ici que les leaders ont une structure de coûts plus avantageuse que les suiveuses. Pour le même prix, la marge de profit est donc plus importante chez les leaders.

8. L'autre possibilité serait de réussir à se reproduire dans le temps, i.e. investir, avec des marges de profit plus faibles que celles des leaders.

9. Même si d'autres définitions du leadership peuvent exister ça et là, Eichner est l'auteur qui a le plus développé la théorie du price leadership. La firme leader est, chez lui, soit la plus grande soit celle qui a la plus grosse part de marché (Eichner, 1976, p : 40). La décision de prix est un acte collectif au sein de l'industrie. Les firmes cherchent à éviter des guerres de prix qui seraient destructrices pour l'ensemble des firmes. En dehors de la collusion explicite qui est prohibée, la coordination collective dans la détermination des prix survient, chez Eichner, du fait que les entreprises s'observent (les déclarations publiques, les comptes rendus d'assemblées générales, les interviews).

10. C'est-à-dire le rapport entre le résultat net et le chiffre d'affaires de l'entreprise : le chiffre d'affaires $(C A)$ est bien le produit du prix de vente $(p)$ par les quantités vendues $(Q)$. Le résultat net $(R N)$ est lui la différence entre l'ensemble des revenus et l'ensemble des charges de l'entreprise, mais, ici, nous l'assimilerons à un résultat d'exploitation, c'est-à-dire à la différence entre le chiffre d'affaires et le coût total $(C T)$. En référence à notre équation (1), il est possible d'exprimer le résultat net de la manière suivante :

$\mathrm{RN}=\mathrm{CA}-\mathrm{CT}=\mathrm{p} \times \mathrm{Q}-\mathrm{CUD} \times \mathrm{Q}=[(1+\theta) \mathrm{CUD}] \times \mathrm{Q}-\mathrm{CUD} \times \mathrm{Q}$

Dès lors, le taux de marge nette (rapport entre résultat net et chiffre d'affaires) que nous obtenons pour nos restaurateurs sera une approximation de leur taux de marge d'exploitation $(\theta)$ :

>> consultez la formule : https://ei.revues.org/5596?file=1

11. L'approche néo-structurale se propose de faire la théorie du phénomène de «coopération entre concurrents » (Lazega, Mounier, 2002 ; Lazega, 2009).

12. Marché d'intérêt national (marché de gros de fruits et légumes).

13. La densité est le nombre de relations observées divisée par le nombre de relations théoriquement possibles si tous les individus étaient en relation. 
14. Il y a réciprocité d'un lien quand un restaurateur cite un autre restaurateur et que ce second restaurateur le cite en retour, ce qui n'est pas systématique.

15. Les données sur la rentabilité étant sensibles, nous n'avons pu les obtenir directement de la part des restaurateurs, nous les avons donc collectées grâce à des fichiers administratifs qui sont consultables gratuitement sur internet: en particulier sur http://www.bilansgratuits.fr/ ou http://www.manageo.fr/. Mais ces fichiers sont lacunaires (notamment pour les restaurateurs constitués en entreprise individuelle). Au final, les données sur la marge nette n'ont pu être recueillies que pour $64 \%$ des restaurants de notre population (soit 200 restaurants sur 314 ).

16. Elle désigne ici la « haute qualité », le « haut de gamme », la restauration dite gastronomique. Ce qui ne sous-entend pas pour autant que les autres types d'établissements soient dénués de «qualités» (telles : la rapidité du service, les prix abordables, le cadre ou concept original, la capacité à accueillir des groupes, etc.).

17. Elle désigne le fait de produire en grande quantité (ici : avoir un grand restaurant pour accueillir beaucoup de clients).

18. Elle désigne le fait de se positionner sur une niche de marché, facilement repérable par les consommateurs comme renvoyant à un type de cuisine particulier mais d'ores et déjà répandu, ce qui la distingue de l'innovation : la spécialisation est une innovation qui a réussi.

19. Puisque le prix est fixé par l'entreprise de manière à couvrir les coûts et à générer un profit, on peut se demander pourquoi certaines entreprises ont une marge nette négative. Ce phénomène peut s'expliquer de deux façons : soit les entrepreneurs ont sous-estimé la marge nécessaire pour couvrir les coûts (plus précisément les coûts fixes) et faire un profit ; soit ils n'ont pas réussi à atteindre le taux de remplissage nécessaire pour générer un profit suffisant. En ce qui concerne la qualité, le problème est que, d'une part l'exigence de qualité gastronomique suppose des coûts, et que, d'autre part, la contrainte marchande impose un prix qui ne peut pas être trop élevé. Autrement dit, ces restaurateurs sont pris entre la nécessité de servir une cuisine de qualité et donc coûteuse, et la contrainte de devoir conserver un prix décent. La manière de résoudre cette double pression est de se servir de la marge de profit comme d'amortisseur. Si l'affluence n'est pas au rendez-vous, le restaurateur finit par réaliser des pertes.

20. Dans ces guides, nous distinguons les récompenses (les étoile au Michelin ou au Bottin Gourmand, les notes au Gault\&Millau, les points au Champerard, les assiettes au Pudlo) des simples citations (qui restent néanmoins des signes de distinction importants).

21. Rappelons que notre population n'a pas été construite pour être représentative, et précisons que cette classe de restaurateurs possédant une «note de qualité » est largement surreprésentée dans notre population finale : nous les avons interrogés de façon quasi-exhaustive.

22. Méthodologiquement, cela implique de transformer le graphe de la figure 1 par une matrice carrée au sein de laquelle tous les individus du réseau sont placés à la fois en ligne et en colonne. À l'intersection des lignes et des colonnes sont placés des « 1 », si une relation existe entre deux individus, ou des « 0 » si cela n'est pas le cas.

23. À l'origine, l'équivalence structurale est une propriété mathématique. Comme, dans la réalité, les véritables cas d'équivalence structurale sont très rares (car deux individus n'ont jamais exactement les mêmes relations), le principe est donc assoupli.

24. Le fait que le regroupement à l'intérieur des blocks ne s'effectue pas sur la base du partage d'attributs sociologiques ou économiques similaires ne signifie pas que ces blocks vont regrouper des acteurs totalement hétérogènes du point de vue des attributs. Le blockmodel regroupe selon les relations interpesonnelles, mais ces relations peuvent au bout du compte nous amener à faire des regroupements d'acteurs originaux, que des regroupements sur la base d'attributs n'auraient pas mis en évidence. Le blockmodel permet ainsi de révéler des communautés sur la base des liens entre ses membres, et ensuite de considérer les attributs partagés par ces membres.

25. On pourra vérifier cette volonté de prétendre, pour les restaurateurs du block $\mathrm{n}^{\circ} 8$, à un prestige qui n'est que virtuel avec les tableaux suivants, notamment les tableaux 5 et 7 . Ces 
restaurateurs se réclament d'une relation avec les restaurateurs gastronomiques du marché, mais proposent une cuisine « industrielle » à leurs clients, et réalisent ainsi des marges de profit élevées en économisant sur les coûts. On a un comportement de type " arriviste ", cherchant à se rapprocher des gens qui comptent dans le métier...

26. Ce n'est pas alors le prix de ces restaurateurs qui est regardé par les autres restaurateurs, mais la qualité de leur cuisine.

27. Cette variable est une donnée déclarative issue de notre enquête. La grande distribution fait référence ici aux grandes surfaces dédiées aux professionnels, comme Metro et Promocash.

\section{RÉSUMÉS}

Cet article aborde la question du leadership sur un marché. Elle mobilise la théorie postkeynésienne et la théorie néo-structurale. La théorie postkeynésienne propose une théorie dite du price leadership selon laquelle un marché serait divisé entre leaders (entreprises dominantes) et suiveurs (entreprises dominées). Cette théorie se heurte à un problème de définition: qu'est-ce qu'un leader dans la théorie postkeynésienne? Est leader celui qui est regardé par les autres répond la sociologie néo-structurale, dont les outils méthodologiques (analyse de réseaux) permettent d'identifier précisément qui est «central » sur un marché. A partir d'un jeu de données empiriques, une enquête de terrain réalisée auprès de trois cents restaurateurs de la métropole lilloise, nous testons la théorie du price leadership. Nous faisons un lien entre le profit économique des entreprises et leur position dans la structure du réseau. Nous montrons que la prise en compte de la seule position structurale ne suffit pas toujours à expliquer la performance économique, mais qu'il convient aussi de prendre en compte le profit symbolique des Chefs cuisiniers comme variable explicative de la position structurale (et de la performance économique). En outre, nous proposons de dépasser le caractère intuitivement individuel de la notion de leadership et montrons l'existence, sur le marché étudié, d'un leadership collectif, à la fois économique et symbolique.

This article deals with the question of leadership on a market. It melts post-keynesian theory and neo-structural theory. Post-keynesian theory puts forward the so-called theory of price leadership according to which a market is divided into leaders (dominant firms) and followers (dominated firms). This theory faces a problem of definition: what is a leader? The leader is the one who is observed by the others: that is the answer of neo-structural sociology, whose methodological tools (networks analysis) allow for precise identifications of who is "central" on a market. Thanks to empirical data based on a field survey led among three hundred restaurants' owners in the surrounding of Lille, we test the theory of price leadership. We establish links between firms' economic profit and their position in the network structure. We show that taking account of the sole structural position is not always enough to explain economic performance, but that it is necessary to also consider symbolic profit of cooking Chefs as explicative variables for structural position (and economic performance). Moreover, we aim at going beyond the intuitive individual property of the notion of leadership, and we show the existence, on the market under study, of a collective leadership, both economic and symbolic. 
INDEX

Mots-clés : marchés, structures sociales, leader en prix, concurrence, marges de profit

Keywords : markets, social structures, price leadership, competition, profit margins

Code JEL B52 - Institutional - Evolutionary, D22 - Firm Behavior: Empirical Analysis, D40 -

General, L10 - General, L20 - General

\section{AUTEURS}

THOMAS DALLERY

Université du Littoral Côte d'Opale, laboratoire TVES

\section{FABIEN ÉLOIRE}

Université Lille 1, laboratoire Clersé

JORDAN MELMIÈS

Université Nice Sophia Antipolis, laboratoire Gredeg 